\title{
COUPLAGES DE RÉSONANCE SYMBIOTIQUE ET HÉTÉRO-ORGANISATION COMME PRINCIPES FONDATEURS DES SYSTÈMES COMPLEXES : ENTRE EFFICACITE DISSIPATIVE ET RÉSILIENCE
}

\author{
Author(s) / Auteur(s) : \\ Jacques de GERLACHE \\ (éco)toxicologue, Dr Sc Pharm. \\ Professeur à l'Institut Supérieur Paul Lambin / General Manager de GreenFacts \\ Membre de l'AFSCET et du groupe Emergence (Paris), Membre du Club de Rome - European Chapter \\ jacquesdegerlache@gmail.com
}

\section{Résumé :}

Il est possible aujourd'hui d'enfin dépasser les limites du paradigme analytique en l'enrichissant d'une réelle dimension systémique permettant une complète intégration des acquis scientifiques récents les plus fondamentaux rendant compte de l'émergence et de la résilience de systèmes complexes, en particulier les états dits "vivants" de la matière. La présente contribution propose de montrer comment l'intégration de cette dimension systémique permet en particulier de mieux comprendre et de gérer de manière réellement lucide et opérationnelle les systèmes d'organisation biologiques et écologiques mais aussi socio-économiques, voire politiques comme, par exemple, en matière de transitions énergétiques face aux bouleversements climatiques.

Les travaux sur la thermodynamique des systèmes loin de l'équilibre ont en effet jeté une lumière sans précédent sur la compréhension de la dynamique des systèmes "complexes", en particulier moléculaires et biologiques. Il est bien établi à présent que l'émergence de telles structures dynamiques d'organisation est déterminée par une maximalisation de leur efficacité dissipative. Celle-ci favorise l'évolution du système au sein duquel elle émerge vers un état d'uniformité (ou d'entropie) maximale décrit par le $2 d$ principe de la thermodynamique. Ces états topologiquement localisés résultant de processus de couplages symbiotiques et "hétéro-organisés" entre composantes distinctes produisant ces états de "résonance dissipative" sont observés depuis le cyclone tropical jusqu'aux systèmes bio-socio-économiques les plus sophistiqués. Il est possible sur cette base d'établir la nature symbiotiques des processus qui déterminent l'organisation et l'évolution des états vivants de la matière moléculaire dans toutes leurs dimensions.

Cela s'est manifesté notamment au niveau organo-biologique par l'émergence de couplages hétéro-organisés et "loca-liés" entre acides nucléiques et acides aminés, ceux-ci donnant naissance aux premières cellules vivantes consommatrices - et donc dissipatives - d'énergie. Des endo-symbioses avec certains organismes unicellulaires primitifs ayant une capacité particulière de capture et de dissipation énergétique, comme les chloroplastes et les mitochondries, autres processus d'hétéro-organisation, ont alors ouvert la voie à l'émergence de structures pluricellulaires co-déterminées encore plus efficaces et résilientes sur le plan dissipatif. Le processus de segmentation (et pas de simple division) d'une cellule "initiale" (l' "œuf") illustre cette co-détermination symbiotique entre composantes épigénétique (le "blanc d'œuf") et génétique (son noyau) ouvrant la voie à l'émergence du potentiel de différenciation tissulaire, y compris dans sa dimension neurologique. Ces processus expliquent l'absence de hiérarchie absolue dans les processus d'hétéro-organisation et leur composante "probabiliste", un peu comme dans un ensemble de jazz chaque musicien peut "improviser" (épigénétiquement) mais en s'accordant en permanence sur les autres dans l'interprétation d'une "composition" (génétique) spécifique.

\footnotetext{
Abstract :

Today it is possible to finally go beyond the limits of the analytical paradigm by enriching it with a real systemic dimension allowing a complete integration of the most fundamental recent scientific achievements reflecting the emergence and resilience of complex systems, in particular the so-called "living" states of matter. This contribution proposes to show how the integration of this systemic dimension makes it possible in particular to better understand and manage in a truly lucid and operational way the biological and ecological organizational systems but also socio-economic, even political as, for example, in terms of energy transitions in the face of climate change.

Work on thermodynamics of far-from-equilibrium systems has indeed thrown unprecedented light on the understanding of the dynamics of "complex" systems, particularly molecular ones. It is now well established that the emergence of such dynamic organizational structures is determined by maximizing their dissipative
} 
efficiency. This promotes the evolution of the system within which it emerges towards a state of maximum uniformity (or entropy) described by the 2 nd principle of thermodynamics. These topologically localized states resulting from symbiotic and "hetero-organized" coupling processes between distinct components producing these "dissipative resonance" states are observed from the tropical cyclone to the most sophisticated bio-socioeconomic systems It is indeed possible on this basis to establish the symbiotic nature of the processes that determine at all levels the organization and evolution of living states of molecular matter.

.This was manifested in particular by the emergence at the organo-biological level of hetero-organized and "localized" couplings between nucleic acids and amino acids, the latter giving rise to the first living cells consuming - and therefore dissipating - energy. Endo-symbiosis with certain primitive single-celled organisms with a particular capacity for energy capture and dissipation, such as chloroplasts and mitochondria, other hetero-organization processes, then opened the way for the emergence of co-determined multicellular structures even more effective and resilient on the dissipative front. The process of segmentation (and not of simple division) of an "initial" cell (the "egg") illustrates this symbiotic co-determination between epigenetic components (the "egg white") and genetics (its nucleus) opening the path to the emergence of the potential for tissue differentiation, including its neurological dimension. These processes explain the absence of an absolute hierarchy in the hetero-organization processes and their "probabilistic" component, a bit like in a jazz ensemble each musician can "improvise" (epigenetically) but by constantly agreeing on the others in the interpretation of a specific (genetic) "composition".

\section{Keywords / Mots-clés :}

thermodynamique, structures dissipatives, systèmes loin de l'équilibre, couplage symbiotique, hétéroorganisation, co-détermination, ago-antagonisme, méthodologie matricielle

\section{Plan du contenu :}

1. "Rupture de causalité" et le concept de "destinée" : par quoi est déterminée l'existence d'un canon?

1.1. Contexte : la crise de la "connai-science"

1.2. (ré)habiliter la notion de cause ultime ou de finalité matérielle dans les causes des phénomènes naturels

2. Combiner réduction et intégration pour transcender enfin la limythe cartésienne

2.1. La matérialité des processus dissipatifs comme explication de l'émergence des états organisés de la matière moléculaire

2.2. Les couplages symbiotiques localisés, moteurs de l'émergence des systèmes dits "organisés"

2.3. Le seuil critique des bifurcations et des transitions de phase

2.4. L'émergence des systèmes dissipatifs cellulaire.

2.5. "ceci n'est pas (seulement) un code" ou des "mystères de la vie" restent à élucider!

3. "pour-quoi il y a des arbres" ou l'émergence des systèmes organiques hétéro-reproductibles

3.1. L'émergence et l'évolution des systèmes dissipatifs multicellulaires : des états végétaux aux états animaux

3.2. L'évolution biologique, entre efficacité et résilience

3.3. Du concept biologique d'auto-organisation à celui d'hétéro-organisation en hypercycles agoantagonistes

3.4. Les causes co-déterminantes d'un phénomène dissipatif moléculaire : une synthèse

4. Transcender enfin les limites du paradigme reduc-tueur de la seule "causalité efficiente"

4.1. Une dimension statistique dans la reproductibilité des organisations hétéro-organisées

4.2. Comment peut s'effondrer un système éco(no)(lo)gique

5. Exemples d'application de l'hetero-organisation systémique: de la gestion d'un orchestre à celle des transitions climatiques et énergétiques

5.1. La connaissance du solfège ne suffit pas à pouvoir coordonner un orchestre

5.2. Une perspective opérationnelle plus intégrative, entre dimensions analytique et systémique

5.3. Développer une structure opérationnelle de projets symbiotiquement hétéro-organisée et intrinsèquement co-déterminée

5.4. Application au cas des projets liés aux transitions des systèmes énergétiques Références 


\title{
1. "RUPTURE DE CAUSALITÉ" ET LE CONCEPT DE "DESTINÉE" : PAR QUOI EST DÉTERMINÉE L'EXISTENCE D'UN CANON ?
}

\author{
"Une idée nouvelle ne triomphe jamais, ce sont ses adversaires \\ qui finissent par mourir."
Max Planck
"Ces mystères qui nous dépassent, feignons d'en être les \\ organisateurs."
}

Jean Cocteau

"Le silence absolu de toutes nos recherches scientifiques à l'égard de nos questions concernant la signification et la portée de la totalité du déploiement apparent est extrêmement pénible."

Erwin Schrödinger, (cité par Etienne Klein)

"Ce n'est pas la manière dont les choses sont réellement qui constitue le problème et qui devrait être changée, mais la prémisse selon laquelle les choses devraient être vues d'une certaine façon."

Paul Watzlawick

"L'homme de science doit faire voir (dessiner) ce que l'on n'a pas encore vu : c'est un concepteur de modèles, de dessins, de théories."

Jean-Louis Le Moigne

"Quand on a compris, on n'a plus besoin de croire."

Albert Constantin

\subsection{Contexte : la crise de la "connai-science"}

Face à la complexité des systèmes que constituent nos sociétés, tant écologiques qu'économiques, et aux crises qui les traversent, ce qui manque toujours le plus aujourd'hui, c'est l'intégration méthodologique rigoureuse des interactions systémiques et irréductibles entre les éléments dynamiques de ces systèmes. Une dimension de l'analyse qui procure pourtant une clair-voyance et donc une lucidité exceptionnelle, quel que soit le domaine auquel on l'applique. La méthode expérimentale alliée à l'outil analytique a certes produit des résultats spectaculaires et a fondé les instruments théoriques capables de décrire derrière leur apparente complexité la simplicité élémentaire de certains processus. Elle a donné naissance à une civilisation technologique inouïe. Néanmoins, quoiqu'obstinément ignorés, les signes de la limite du pouvoir explicatif du principe réducteur de la logique causale se manifestent de plus en plus, et ce jusqu'aux niveaux sociétal et environnemental, particulièrement irréductibles. Du fait de l'asservissement à la contrainte idéologique d'une logique purement réductrice et déterministe et à celle de la causalité linéaire, la rationalité s'est muée progressivement au plan scientifique puis philosophique et socio-politique en un 'rational-isme' dogmatique, alors que l'approche réductive et son outil conceptuel qu'est la logique déductive se sont transformés en 'réduction-nisme'. Le chaînon systémique permet pourtant de mieux décrire comment et pour-quoi des "parties" peuvent dans certaines circonstances s'assembler pour produire un effet, une structure, un état émergent, bref un "tout", ni réductible, ni déductible du seul et strict ensemble de propriétés de ses parties individuelles.

\section{2. (Ré)habiliter la notion de 'cause ultime' ou de 'finalité matérielle' dans les causes des phénomènes naturels.}

Le problème est que, comme tous les humains, et même face à l'évidence de nouvelles faits établis et relatifs à la compréhension de la réalité qui nous entoure, les scientifiques restent souvent attachés à leurs représentations paradigmatiques, voire "mythiques", qu'ils se sont construits de la réalité. Cinq 
siècles après Nicolas Copernic, au lever du jour, la plupart voient toujours le soleil "monter sur l'horizon", sachant pourtant que c'est l'inverse de la "réalité" !

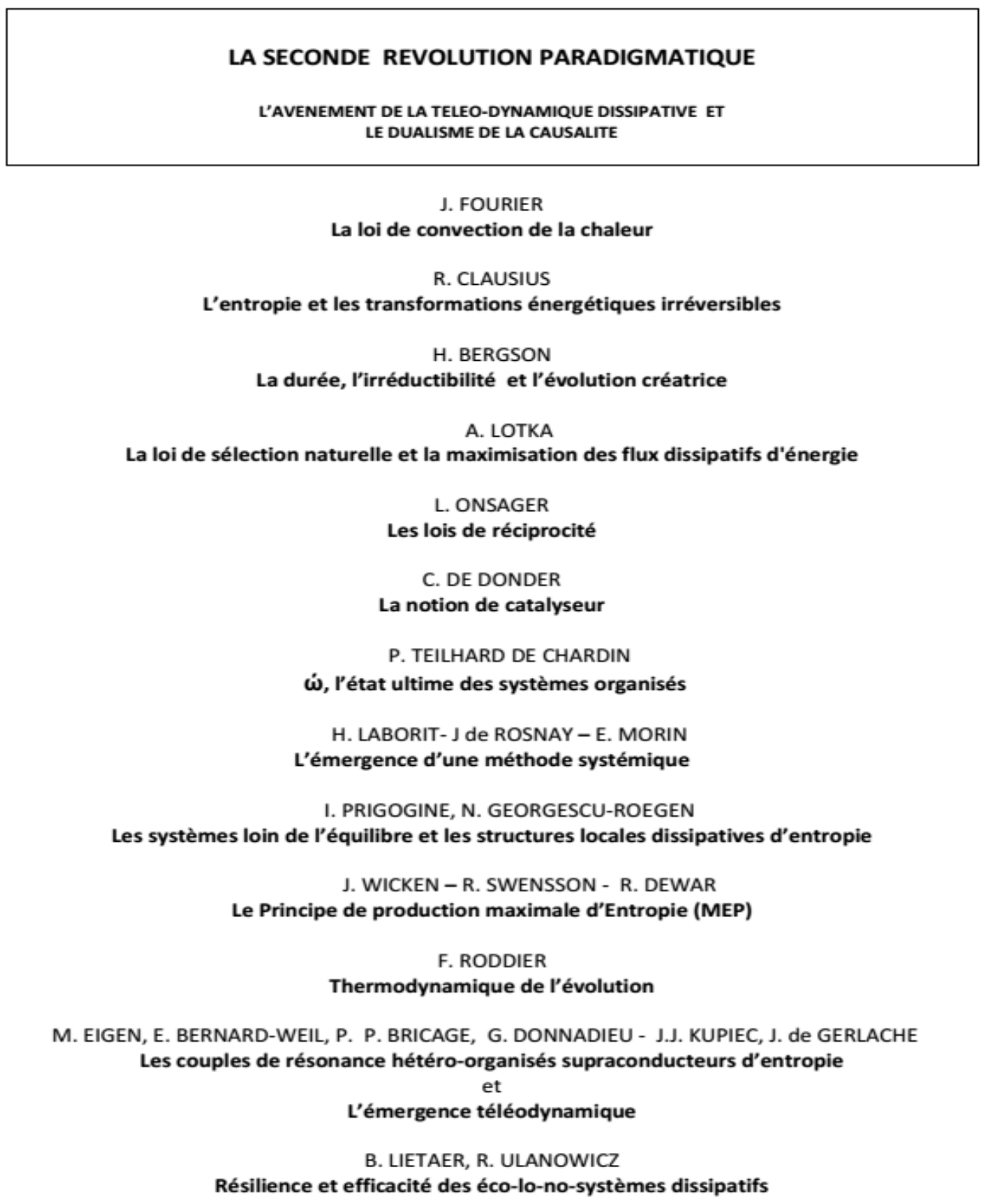

C'est en particulier sous prétexte d'éviter tout retour d'un finalisme teinté de métaphysique, que s'est imposé le paradigme post-cartésien de la réversibilité intemporelle des phénomènes observables expérimentalement, vision qui serait incontournable pour qu'un phénomène puisse être considéré comme objectivable "scientifiquement". René Magritte nous a pourtant expliqué que "Ceci n'est pas une pomme" : la représentation que chacun s'en fait n'est jamais vrai-ment une pomme. Mais "prendre de la hauteur" pour accepter de "regarder au-delà" de nos limites paradigmatiques de représentation, il 
faut vraiment le vouloir et le pouvoir ! Tant qu'il s'agit d'un poétique lever du jour, soit ; mais quand il s'agit de "voir" et d'accepter de "regarder au-delà" la réalité des processus physico-chimiques, biologiques et écologiques, voire politiques et socio-économiques parmi les plus essence-tiels et tels que de nouveaux éclairages nous les révèlent, c'est tout autre chose (de Gerlache, 1994, Kaufman, 2006).

En biologie, par exemple, le réductionnisme moléculaire fut au cœur du paradigme de la biologie des années 1960-90 au travers d'une vision très informaticienne, qui était en plein développement à la même époque, de stricts "programmes génétiques" et cela malgré l'émergence conjointe de la science cybernétique. Il demeure encore vivace alors qu'aucune démonstration expérimentale, voire clinique, directe ne vient pourtant en démontrer la conformité universelle face à la réalité des processus systémiques d'organisation observés dans les systèmes biologiques. Cette vision réductrice a notamment dominé pendant plus de 30 ans la cancérologie au travers de la recherche de "la" mutation spécifique hypothétique qui serait la cause d'un cancer. Pour certains groupes de chercheurs, il fut difficile de "survivre" dans ce paradigme dominant (de Gerlache \& Lans, 1983, de Gerlache et coll, 1984, 1986).

Emmanuel Farber (1984) et Harry Rubin (1992) avaient été parmi les premiers à suggérer que le cancer est une maladie épigénétique qui ne serait pas directement ou totalement liée à une altération de l'ADN génomique, concept repris notamment par Mae-Wan Ho (1998). Comme expliqué par Ho, les tumeurs ont plus d'une centaine de mutations génétiques dans les régions codantes seules, et non seulement les mutations observées chez les patients individuels diffèrent les unes des autres, mais les différentes régions d'une même tumeur ont également des profils génétiques nettement différents. C'est précisément l'image à laquelle on pourrait s'attendre si les mutations génétiques sont les effets plutôt que les causes du cancer; les cellules tumorales émergeant étant des cellules dont des mutations géniques, grâce à une "fluidité" du génome observée dès la fin des années 70 , leur permettent de survivre en échappant aux contraintes symbiotiques de l'organisme au détriment du patient. Ce qui mena Robert Weinberg, checheur éminent qui avait identifié plusieurs "oncogènes", à faire dans des articles à présent célèbres avec son collègue Douglas Hanahan (2000, 2010), un examen critique des limites devenues avérées de ce paradigme en affirmant :

"Deux notions, qu'un simple petit nombre de gènes suffirait pour transformer des cellules en cellules cancéreuses, et que toutes les cellules de mammifères suivraient le même ensemble de processus génétiques au cours de la transformation néoplasique ont été mis en cause. (...) J'en suis venu à comprendre que les opinions bien ancrées de professeurs de haut niveau largement respectés doivent être pris avec de grosses pincettes."

Ils poursuivent en affirmant: "La relation entre les données expérimentales et leur pertinence biologique s'est effiloché, si pas cassé. Nous n'avons pas les paradigmes conceptuels et des stratégies informatiques pour faire face à cette complexité." (!!!)

"Et tout aussi douloureux, nous ne savons pas comment intégrer les ensembles de données individuelles, comme celles découlant de génome des cancers analysés, avec d'autres ensembles de données tout aussi importants, tels ceux de la protéonomique." (...)

"Le concept qui s'impose à présent est qu'un système complexe ne peut être compris que si l'ensemble de ses pièces sont analysées dans un vaste panorama d'ensemble. Ces analyses globales devraient idéalement décrire la réalité complexe des systèmes biologiques réels, y compris celle des cellules cancéreuses." 


\title{
2. COMBINER RÉDUCTION ET INTÉGRATION POUR TRANSCENDER ENFIN LA LIMYTHE CARTÉSIENNE
}

\author{
"La simplification des connaissances mutile la réalité" \\ "A partir du moment où la démarche de décomposition \\ Edgar Morin \\ analytique et de reconstruction synthétique ne permet plus de \\ retrouver les propriétés du tout, elle perd son pouvoir \\ explicatif et devient pratiquement inutile". \\ Pierre Delattre, CEA \\ "C'est le contraste de plus en plus évident, de plus en plus \\ difficile à dissimuler, entre une science pléthorique et la \\ stagnation manifeste de la pensée scientifique vis-à-vis des \\ problèmes centraux qui affectent notre connaissance de la \\ réalité." \\ René Thom (cité par J.L. Le Moigne) \\ "J'ai essayé pendant ma vie de bâtir une science du devenir. Et \\ une science du devenir est une science en devenir, encore \\ imparfaite."
}

Ilya Prigogine

\subsection{La matérialité des processus dissipatifs comme explication de l'émergence des états organisés de la matière moléculaire}

L'exemple le plus élémentaire de système émergent est sans doute celui de la vidange d'un évier dont le tourbillon convectif, en organisant le mouvement cohérent de milliards de molécules d'eau, en favorise spontanément le processus. Dans un tel ensemble d'éléments matériels, localement uniformes et désordonnés mais loin de l'équilibre, une convection s'installe lorsque des interactions dynamiques entre ces éléments concourent à l'émergence d'un mouvement d'ensemble cohérent. Le tourbillon, dont la cohérence "transcende" celle que les éléments ont individuellement, se crée et le liquide s'écoule alors avec une "efficacité" amplifiée et cet état d'organisation stationnaire est spontanément "conservé" tant qu'il contribue à l'évolution du système global vers un état d'équilibre.

Cette propriété structurante de molécules est déjà décrite par Tyndall (1869) avec de simples grains de sable placés sur une plaque en vibration. Des modes de résonance oscillants vont induire la formation de motifs géométriques via un afflux d'énergie qui ne modifie pas leur énergie propre mais la configuration globale pour former un motif macroscopique "cohérent".

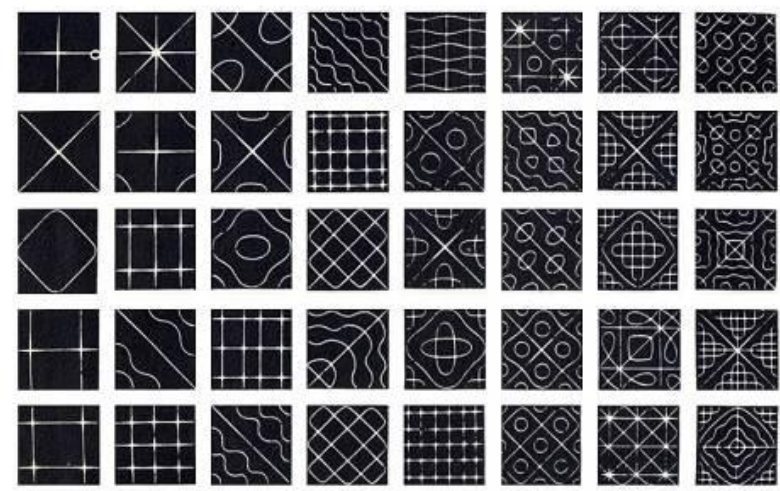

\footnotetext{
Modèles de résonance vibratoire de Chladni. Les zones sombres désignent des régions de vibrations intenses (fortes fluctuations isotropes), qui sont liées par des lignes lumineuses avec de faibles fluctuations isotropes (lignes nodales de vibration nulle).
} 
Trois éléments sont déterminants dans l'analyse des principes gouvernant l'apparence de tels processus :

1. les "causes initiales" déterminant le potentiel d'apparition du phénomène ;

2. les "contraintes locales" endogènes au système et déterminant son émergence ;

3. les "causes ultimes" déterminant le maintien, voire l'évolution du phénomène.

A la causalité initiale efficiente, endogène, rendant compte de l'apparition du phénomène, se combine en effet localement une causalité exogène et "ultimisante" (pour ne pas dire "finalisante" ..."), celle qu'impose la contrainte décrite par le second principe de la thermodynamique : l'évolution d'un système vers un état d'équilibre caractérisé par son état d'uniformité énergétique maximale. Il y a donc là une combinaison de causalités qui induit ce qui sera appelé une transition de phase dans lesquelles les contraintes imposées aux "parties" deviennent localement subsidiaires des contraintes imposées à l'ensemble de leurs interactions et qui déterminent l'émergence du "tout", en l'occurrence le système tourbillonnaire. C'est une "raison d'état thermodynamique" qui contribue ainsi à l'émergence de cette transition de phase. La Seine à Paris, entre sa source et son ultime estuaire comme "causes déterminantes", n'est-elle pas une image d'un tel état dynamique de 'conatus symbiotique' dans une 'Nature unique' évoquée par Bento Spinoza ... ?

A remarquer que les œuvres-clés de René Magritte (encore lui, le contemporain de la naissance de la systémique et de la thermodynamique dissipative), suscitent des états mentaux d'émergence en créant de telles symbioses dynamiques entre les "objets" de ses tableaux : la nuit et le jour coexistant dans ' $L$ ' Empire des lumières', le tableau intégré dans le paysage de 'La Condition humaine', etc, ...

Si les travaux de théoriciens et de praticiens de la complexité comme Norbert Wiener (1948), Herman von Foerster, John Holland, Paul Watzlawick, Ludwig von Bertalanffy, Axel Kleidon, et, en Europe, notamment Edgar Morin, Jean-Louis Le Moigne, Gérard Donnadieu, Joël de Rosnay, François Dubois, Gérard Balantzian, Henri Atlan, Jacques Printz, Pierre Massotte et Patrick Corsi et bien d'autres encore, au sein notamment des groupes de l'Afscet et du Groupe Emergence ${ }^{1}$, ont décrit les états et certaines des conditions devant être réunies pour générer de telles émergences, la thermodynamique dissipative sous la houlette notamment de Ilya Prigogine et ses collaborateurs (1969a, 1969b, 1970) mais aussi de Nicholas Georgescu-Roegen (1979), a permis d'identifier et de caractériser de façon beaucoup plus précise et opérationnelle les conditions thermodynamiques propices à ces processus d'émergence d'états d'organisation complexe de la matière, en particulier moléculaire. Ce qui nous amènera notamment au concept de "résonances convectives".

Comme le souligne Gérard Donnadieu (Donnadieu et Karsky, 2002), aussi dans ses "Repères historiques de la science des systèmes" (2004), Norbert Wiener et ses amis publient en 1943 un manifeste "anti-positiviste" récusant le déterminisme et en restaurant le principe de finalité, véritable coup de force qui est passé alors pratiquement inaperçu de la plupart des scientifiques. D'une manière générale, Il est frappant de constater que tant de physiciens intéressés par l'examen des propriétés de la matière aux niveaux de ses dimensions particulaire ou sidérale semblent toujours négliger un peu la réalité du niveau (macro)moléculaire qui est pourtant celui qui définit si largement leur propre réalité...

Or il est temps d'intégrer plus complètement le fait que cette dimension moléculaire, et donc "chimique", du réel induit des propriétés fondatrices qui lui sont propres, entre les deux autres dimensions. C'est parce qu'elle est également co-déterminée par ces autres dimensions que l'analyse et la compréhension de la réalité de la dimension moléculaire impose une méthode intégrative dont on comprend alors la nature complexe et irréductible. Cette dimension moléculaire est sans doute actuellement la mieux appréhendée par la mécanique statistique, l'autre appellation de la "thermodynamique". C'est la discipline de la science dont Albert Einstein lui-même affirmait être convaincu qu'elle est : "la science naturelle d'une portée universelle qui, dans le champ de l'applicabilité de ses concepts de base, ne sera jamais réfutée." Au niveau planétaire, la nature des processus thermodynamiques est déterminante par le fait que la destinée de ce système ouvert est

\footnotetext{
${ }^{1}$ http://www.mountvernon.fr/qui_sommes_nous.html
} 
d'évoluer spontanément vers un état d'uniformité ultime de la répartition de son énergie : qu'elle soit d'origine tellurique, solaire, cosmique, physico-chimique ou ... biologique.

Les structures dissipatives localisées émergent comme des "systèmes dynamiques organisés" à relativement plus faible niveau d'entropie que celui du milieu global au sein duquel ils émergent et résultent de couplages symbiotiques dont l'effet de "résonance" produit une forme d'effet "supraconducteur d'entropie".

Un (éco)système organisé est donc de l'ordre qui émerge localement pour favoriser l'uniformité ou dés-ordre global :

- d'une part, via les processus énergétiques qui contribuent à l'augmentation locale de l'ordre et la contrainte dans les systèmes vivants ;

- d'autre part, via les processus maximalisés de dissipation de cette énergie, et donc d'évolution vers une uniformité dés-ordonnée du système global, telle que décrite par la seconde loi de la thermodynamique.

N'est-ce pas ce que reflète l'intuition de la vision "philosophique" extrême-orientale qui, dans la notion de yin-yang, combine en un "pile $\underline{e t}$ face", plutôt que de les opposer, les deux dimensions de la réalité des systèmes matériels organisés?

\section{Complexité néguentropique}

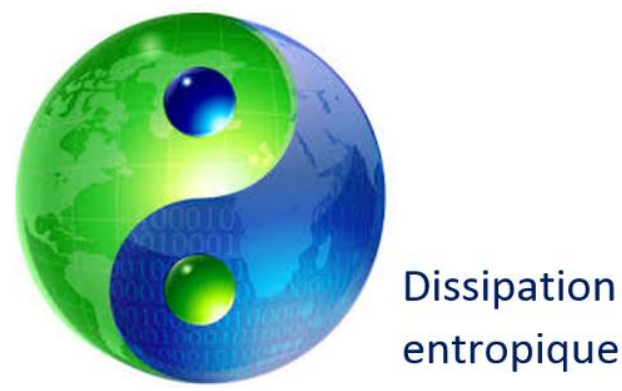

Dans un tel contexte, l'équilibre thermique de la planète Terre est déterminé par des systèmes (thermo)dynamiques "localisés" qui maximalisent la dissipation de son énergie en uniformisant sa répartition. Ce sont notamment les grands courants atmosphériques (cyclones et anticyclones) et marins qui maximalisent les échanges thermiques tout en étant soumis à des boucles de rétroaction positives et négatives ayant des constantes de temps très différentes les unes des autres ${ }^{2}$ : c'est leur "raison d'être" !

Cette évolution vers l'uniformité énergétique est donc une forme de "destinée" nullement "intentionnelle" et constitue un attracteur qui, "finalement" n'est pas aussi étrange que cela...

\footnotetext{
${ }^{2}$ D'autres facteurs interviennent, évidemment, comme le fameux "effet de serre".
} 


\title{
2.2. Les couplages symbiotiques localisés, moteurs de l'émergence des systèmes dits "organisés"
}

\begin{abstract}
"Les cellules ne se ressemblent-elles pas comme des molécules, plus que comme des animaux ?... Nous les regardons à bon droit comme les premières des formes vivantes. Mais n'est-il pas juste aussi vrai de les considérer comme représentant un autre état de la Matière : quelque chose d'aussi original, dans son ordre, que l'électronique, l'atomique, le cristallin ou le polymère?

Un type nouveau de matériel, pour un nouvel étage de l'Univers?"

Pierre Teilhard de Chardin

"L'auto-organisation, l'autoréférence, l'autogenèse, l'autopoièse sont des termes qui ont trop longtemps polarisé les chercheurs et ils n'ont de sens que couplés, comme nous l'avons proposé dans les années 80 , avec, respectivement ce que nous appelons l'hétéroorganisation, l'hétéroréférence, l'hétérogenèse, l'hétéropoièse [de même que nous ayons insisté sur le mésusage du terme d'émergence qui, lui aussi, doit être couplé avec celui d'immergence (ou d'immersion)."
\end{abstract}

Elie Bernard-Weil

Il s'agit à présent d'établir comment et pour-quoi au sein du système terrestre, des structures organisées particulières, les structures organo-biologiques, dites "vivantes" c'est-à-dire "dynamiquement résilientes et adaptatives", vont émerger loin de l'équilibre.

Ce qui caractérise de telles structures, c'est le fait à présent bien établi que, dans sa phase de croissance et de maturité, un organisme vivant est dans un état dynamique stationnaire caractérisé par un taux maximal de production et de dissipation d'entropie (Lotka, (1922a, 1922b), Wicken (1987), Swenson, (1988, 1989), Dewar (2003). Cet état particulier, qui n'a étonnament pas été vraiment été étudié plus en détail par Prigogine et Georgescu-Roegen cités précédemment, étant favorisé et "sélectionné" par le fait même qu'il contribue à ce que le système global loin de l'équilibre au sein duquel émergent ces structures de maximalisation dissipative, atteigne le plus rapidement et le plus efficacement possible son état d'équilibre et d'entropie maximale déjà évoqué, conformément à la seconde loi de la thermodynamique.

Comme l'a exprimé Rod Swanson (1991), "la production d'ordre est inexorable parce l'ordre favorise la production d'entropie plus que le désordre et l'émergence progressive de cycles perception-action dans l'évolution de la Terre en tant qu'entité globale est le produit licite de la physique opportuniste : il n'y a pas d'autre moyen de produire les états collectifs (ordonnés) qui engendreraient ces niveaux plus élevés de dissipation."

Si on analyse les systèmes dissipatifs les plus simples comme un tourbillon ou le cyclone déjà évoqués, on observe que leur moteur réside dans un couplage dissipatif et localisé de deux forces ou flux. Pour le cyclone, en raison de la rotation de la Terre, une force de déviation circulaire, dite force de Coriolis, s'exerce sur les corps, y compris l'air en mouvement à la surface du globe et de ses océans ; les flux ascendants de vapeur d'eau chauffée commencent alors à tourbillonner. Dès lors, les vents s'intensifient, l'évaporation s'accentue. Au-delà d'un seuil critique de la température de l'eau $\left(27^{\circ} \mathrm{C}\right)$ constituant une bifurcation ou transition de phase, un immense tourbillon se crée et le cyclone forme. En altitude, la chaleur se dissipant, les nuages se forment car la vapeur refroidie se recondense. Dans le cas du tourbillon dans un évier, c'est la forme de celui-ci qui va contribuer à amorcer le mouvement de convection en combinaison symbiotique avec la force attractive de la "pesanteur".

Ainsi l'émergence d'un système dissipatif organisé et structuré au sein d'un tel système est bien codéterminée à la fois par des "causes initiales" et par une "cause ultime", cette destinée décrite par la loi 
entropique de dissipation des déséquilibres énergétiques. Ces états résultant d'une véritable "combinaison symbiotique" localisée et "organisée" d'éléments moléculaires en interaction(s). Ici encore on peut évoquer le cours d'un fleuve, co-déterminé à la fois par sa source et par sa destinée vers l'océan, qui n'est donc pas totalement déterminée par sa source.

Un phénomène d'organisation moléculaire dissipative comparable à celui d'un cyclone mais observable à beaucoup plus petite échelle est celui du phénomène des cellules de Bénard-Rayleigh qui apparaissent spontanément dans un liquide quand on lui applique une source de chaleur extérieure (Bénard, 1901). On dépose une couche de liquide, par exemple de l'eau ou un liquide un peu plus visqueux, dans une boite de Pétri que l'on chauffe par le bas. Le flux d'énergie va d'abord se dissiper verticalement au travers du liquide par conduction thermique, selon un gradient thermique linéaire décrit par la seconde loi de la thermodynamique. Si la température de la face inférieure continue à croître, au-delà d'un certain seuil apparaîtront des cellules de convection, micro-cyclones disposés comme les cellules dans un nid d'abeilles. Les cellules de convection sont stables, et leur sens de rotation, inverse pour deux cellules contigües dans le sens horizontal vont maximalise la dissipation de la chaleur au sein du liquide.

Un autre phénomène simple de couplage dissipatif qui sera par la suite associé à des propriétés d'affinité entre composants des systèmes biologiques, est observé avec des molécules organiques : c'est la réaction mise en évidence par Boris Belousov (1950) et confirmée ensuite par Anathol Zhabotinski. Des ions bromate $\mathrm{BrO}^{3-}$, de l'acide citrique ou maléique, en présence d'ions cériques $\mathrm{Ce}^{4+}$, qui jouent le rôle de catalyseur, sont mélangés dans une boite de Pétri à température ambiante. Ces composants sont proches de ceux impliqués dans le cycle dit de Krebs d'oxydation cellulaire. A l'étonnement même de Boris Belousov, la solution oscille alors entre deux états, dont l'évolution est périodique et qui apparaissent en surface comme des ondes de concentration et ce, pendant près d'une centaine de cycles, jusqu'à épuisement d'un des réactifs.
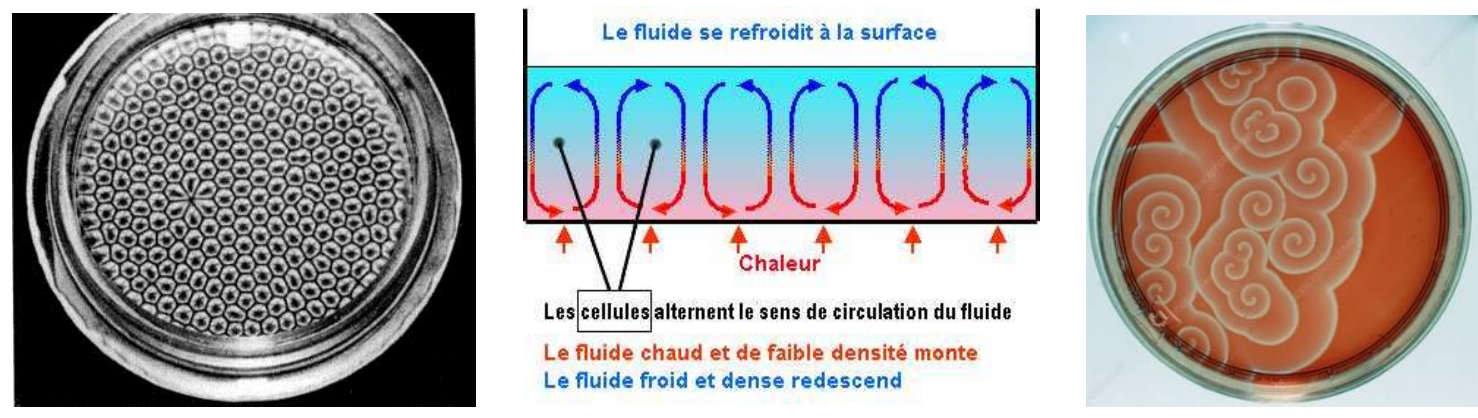

A gauche : des cellules de Bénard : schéma créé par Thérésa Knott, traduit et modifié pour wiki fr https://fr.wikipedia.org/wiki/Cellules_de_B\%C3\%A9nard\#/media/Fichier:Cellules_de_Bénard.jpg A droite : effet de diffusion dissipative de Belousov et Zhabotinski : https://media.sciencephoto.com/image/a5000005/400wm/A5000005Belousov-Zhabotinsky_reaction_in_dish.jpg

Soumis à une première revue, le rapporteur en refusa les résultats, déclarant que cette expérience allait à l'encontre du deuxième principe de la thermodynamique. Un second refus de publication dans un autre périodique fut enregistré six mois plus tard et Belousov ne parvint à partager ses travaux que dans le compte-rendu d'une conférence qu'il donna en ... 1958 ! En 1964, Zhabotinski (1964) reprit l'expérience réalisée par Belousov qui en fut informé mais refusa de le rencontrer avant de mourir en $1970 \ldots$ Le prix Lénine leur fut finalement attribué conjointement en 1980.

Alfred Lotka (1922a, 1922b) avait été le premier à formuler explicitement le fait que les organismes vivants "maximisent le flux d'énergie qui les traverse". Dans ses articles de 1922, il considéra que la force motrice de la sélection naturelle est la maximisation des flux d'énergie par l'organisme à condition qu'il y ait toujours un résidu non utilisé de l'énergie laissée dans le système. Il affirma dès lors que la sélection naturelle fonctionne comme réponse à une 3ème loi de la thermodynamique en favorisant les organismes les plus aptes à survivre et à se reproduire, cette sélection maximisant le taux global de dissipation de l'énergie. Reconnu aussi à titre posthume pour sa fameuse formulation 
mathématique des lois de la dynamique des populations (loi de Lotka-Volterra, (Lotka, 1925), il fut à l'époque, comme Belousov plus tard, rejeté par le dogmatisme de ses pairs et dut se résoudre de 1924 à sa retraite à travailler en tant que statisticien pour ... une compagnie d'assurances ! Il faudra attendre Jeffrey Wicken (1987), Rod Swanson (1988, 1989), Roderick Dewar (2003), Per Bak (1996) et quelques autres sur le chemin ouvert par Théophile De Donder ${ }^{3}$ et Ilya Prigogine, avant que cette notion ne prenne tout son sens au travers des sciences, biologiques en particulier, et la formulation de ce qui constitue à présent une 3ème loi de la thermodynamique, dite de production maximale d'entropie (Maximal Entropy Production ou "MaxEP" (voir Roddier, 2012).

\subsection{Le seuil critique des bifurcations et des transitions de phase}

Le processus fondamental dans l'émergence d'une structure dissipative moléculaire est donc l'établissement, à un seuil critique, d'une "transition de phase", une "bifurcation" établissant localement dans un ensemble d'éléments des couplages localisé de forces au sein d'un flux entropique. Selon Prigogine (1980), une nature en bifurcation est celle où de petites différences, des fluctuations insignifiantes, peuvent, si elles se produisent dans des circonstances opportunes, envahir tout le système, engendrer un régime de fonctionnement nouveau. Les bifurcations sont alors "des points singuliers où une branche se subdivise en plusieurs branches ou même en un nombre infinis de branches. Et le choix de la branche qui sera suivie dépend des fluctuations. ( ...) Entre les points de bifurcation, le déterminisme n'est qu'une approximation (...) tandis qu'aux points de bifurcation, vous n'avez plus d'approximation déterministe". La complexité observée émerge alors d'une manière robuste qui ne dépend pas de détails précis du système : les paramètres variables du modèle peuvent être largement modifiés sans affecter l'émergence d'un comportement critique (d'où l'appellation "criticité auto-organisée"). Ce qui constitue la découverte d'un mécanisme par lequel l'émergence de la complexité à partir de simples interactions locales pourrait être spontanée et donc une source plausible de complexité naturelle.

Spécialiste des "états critiques" de la matière, le physicien danois Per Bak (1996) a montré que certaines structures dissipatives évoluent de façon à toujours se rapprocher d'un point de bifurcation. En ce point, la structure dissipative est dans un état "critique" correspondant pour un liquide à une certaine température et une certaine pression où un fluide homogène peut se décomposer en deux "phases", une phase liquide et une phase vapeur. Bien que le fluide soit en équilibre thermodynamique, ce processus est formellement équivalent à celui d'une bifurcation d'une structure dissipative (les équations sont analogues).

Selon Per Bak, l'une des particularités des systèmes auto-organisés à l'état critique est alors de posséder une double signature fractale: temporelle et spatiale. Les variables qui décrivent le comportement du système suivent des lois puissance et les systèmes auto-organisés critiques construisent ces formes fractales. Il décrit une suite d'états critiques où des "bouffées d'énergie" se libèrent - comme dans le cas des avalanches ou des tremblements de terre - bouffées d'autant plus fréquentes que leur amplitude est plus faible. C'est ce que les physiciens appellent un "bruit en 1/f". Son importance vient du fait qu'on les observe très souvent, non seulement en électronique, mais aussi en astronomie, en géophysique, en biologie (nous y reviendrons) et même en économie.

Les structures dissipatives s'adaptent donc à partir des fluctuations perçues de l'environnement par les suites d'avalanches consécutives à l'établissement et l'accumulation de relations couplées (ce que l'on nomme feedback positif et négatif d'information en cybernétique) entre leurs éléments constitutifs. C'est le processus qui détermine par exemple la stabilité des écosystèmes, comme l'illustre l'exemple d'un château de cartes: on peut enlever une, deux ou plusieurs cartes (ou espèces) sans grande conséquence pour le système. Mais soudain enlever une seule carte supplémentaire provoque l'écroulement du château : l'écosystème disparaît ... Et ce sera peut-être bientôt un tel point de rupture

\footnotetext{
${ }^{3}$ Professeur à l'Université de Bruxelles, Il a développé en 1923 la définition de l'affinité chimique en soulignant un lien entre affinité chimique et l'énergie libre de Gibbs. Inspirateur de Ilya Prigogine, Il est aussi considéré comme le père de la thermodynamique des processus irréversibles.
} 
de l'équilibre climatique que rencontrera notre planète toute entière, bien loin des scénarios plus ou moins linéaires que l'on nous délivre généralement ${ }^{4} \ldots$

Etudiant la dynamique de réseaux d'automates cellulaires engendrés d'une façon aléatoire, Stuart Kaufman (1993, 1995) a observé que des formes stables animées d'un mouvement périodique apparaissaient spontanément, quelles que soient les conditions initiales, générant des formes complexes et durables engendrées d'une façon purement aléatoire. Il a mis, ici encore, quelques années avant de le faire accepter par d'autres scientifiques. Selon Kaufman et Johnsen (1991), une coévolution est considérée, au niveau le plus simple, comme un couplage de paysages de telle sorte que les mouvements adaptatifs d'un acteur déforment les paysages, la robustesse des paysages, la richesse de l'interconnexion de deux paysages et le nombre d'autres acteurs qui interagissent avec chaque acteur.

Toutes ces propriétés modifient profondément le caractère de la dynamique co-évolutionnaire et régissent la facilité avec laquelle les écosystèmes en coévolution atteignent les équilibres de Nash, la stabilité aux perturbations de tels équilibres et la forme physique moyenne conservée des partenaires en co-évolution. Ayant compris que ces observations renouvellent d'une façon très originale l'explication des phénomènes liés à la question de l'origine de la vie, ils ont suggéré la possibilité qu'une méta-dynamique évolutionnaire due à la sélection naturelle puisse alors sculpter les paysages et leurs couplages pour obtenir des systèmes co-évolutionnaires capables d'adaptation.

\subsection{L'émergence des systèmes dissipatifs cellulaires}

Comme les cyclones, les cellules bactériennes sont des machines de dissipation thermique. Durant des millions d'années en effet, au hasard de combinaisons chimiques, ont émergé des ensembles (macro)moléculaires protocellulaires, peut-être des sortes de vésicules aplaties (les "obcells"5) avec leur matériel génétique et leurs enzymes ancrés sur leur face externe, capables de produire localement de tels "cycles dissipatifs d'énergie". Initialement ces "bulles" qui se seraient formées spontanément et qui ont constitué la structure de base des membranes cellulaires, sont apparues sans doute dans le sillage des sources géothermales du fond des océans, à partir de molécules organiques ${ }^{6}$ (notamment des phospholipides et des peptidoglycans), voire des "microbes" d'origine cosmique (Steel et coll., 2018). Des acides aminés et des bases nucléiques ont en effet été identifiés dans des météorites à chondrite carbonée, supposées être dérivées de comètes, telles que le météorite de Murchinson. La première véritable cellule dite vivante serait née de la fusion de plusieurs de ces "obcellules" en agrégat.

Dans ces cellules, ce sont des relations catalytiques qui abaissent la barrière énergétique de certaines réactions chimiques entre molécules organiques ayant des affinités structurelles entre elles (les "enzymes") et amplifient par ce fait même l'efficacité du processus dissipatif. Dans un tel milieu "cellulaire", et comme observé déjà dans la réaction de Belousov-Zhabotinski, les produits des réactions catalytiques vont alors diffuser et générer d'autres couplages de résonance 'supraconducteurs d'entropie'. Ceci tout en induisant une résilience de ces états dissipatifs via l'apparition de processus d'actions et rétroactions stabilisateurs, tel l'effet "feed-back" dans une simple chasse d'eau.

Le modèle proposé de son côté par Alan Turing (1954) expliquait déjà la morphogénèse en biochimie par le phénomène spontané de "brisure spontanée de symétrie". La dynamique résulte du couplage entre des réactions chimiques et la diffusion des réactifs, dans un système alimenté en continu. Turing parle d'"ondes stationnaires" pour désigner les motifs, mais le terme ne doit pas prêter à confusion avec les équations d'onde décrivant la propagation du son ou de la lumière. Elles

\footnotetext{
${ }^{4}$ https://www.thethirdpole.net/en/2017/11/17/earth-approaching-tipping-point-warn-scientists/ http://www.metlink.org/climate/ipcc-updates-for-a-level-geography/tipping-points/ https://www.dunod.com/sciences-techniques/mettre-en-oeuvre-transitions-energetiques-strategie-integrative-et-gestion ${ }^{5}$ https://en.wikipedia.org/wiki/Obcell

${ }^{6}$ Les expériences de S. Miller S. et H. Urey (1959) ont démontré à partir d'eau, de méthane, d'ammoniac et d'hydrogène, la synthèse de 13 des 22 acides aminés utilisés dans la synthèse des protéines, des sucres, des lipides, et quelques composants des acides nucléiques.
} 
correspondent aux variations spatialement périodiques des concentrations des espèces chimiques et, précise-t-il, ne sont rien d'autre qu'un phénomène de résonance, bien connu dans l'étude des pendules et autres oscillateurs, la solution de l'équation d'évolution ne répondant à aucun programme préalable, ni "prescription spécifique" extérieure. L'échelle spatiale des motifs est sans commune mesure avec les échelles moléculaires des processus sous-jacents, montrant le caractère émergent de leur formation. Peut-on parler ici encore de "déterminisme" au sens "étroit" du terme ? On retrouve ici le rôle du métabolisme à côté du génétique et celui le concept d'autopoièse ${ }^{7}$ développé par Varela, Maturana et Uribe (1979), qui s'inspire de celui des systèmes dissipatifs. Nous y reviendrons en évoquant les processus épigénétiques liés au développement embryonnaire.

René Lefever et Ilya Prigogine (1968) calculèrent de leur côté les conditions d'une distribution stable pour un système de réactions chimiques à "deux boites" (le "Brusselator") et démontrèrent les capacités "morphogénétiques" des processus de couplage chimio-diffusifs. Si les frontières du système sont maintenues constantes, sous l'influence d'un courant (flux) thermodynamique, le système évolue spontanément vers un état d'équilibre dynamique stationnaire. La structure dissipative est donc ici encore décrite comme une structure bipolaire co-déterminée par le biais de relations catalytiques entre ses éléments constitutifs qui ont la propriété d'abaisser la barrière des échanges énergétiques entre molécules ("enzymes") en ayant entre elles des affinités spatiales, dites allostériques. Certaines de ces structures allostériques auront en outre la capacité de reproduction par empreinte de leur propre structure, cette propriété qui caractérise les états dissipatifs qualifiés des états "vivants" de la matière. Cette propriété catalytique étant le fondement de leur capacité à maintenir leur propriété "supraconductive" de dissipation.

Ce sont probablement des molécules d'acide ribonucléique primitif captées par les microbulles bulles de lipides précédemment évoquées qui, absorbant efficacement les photons dans la région ultraviolette à 200-300 nm du spectre solaire, ont commencé à capter et à dissiper par effets de résonance l'énergie disponible. Par exemple, des types "antenne" de molécules d'acides nucléiques pourraient, par transfert de l'énergie d'excitation électronique captée par résonance, utiliser leurs intersections coniques pour dissiper rapidement cette énergie induite par les photons en chaleur (Michaelian, 2011, 2016), La polymérisation spontanée sous lumière UV de nucléosides en polynucléotides a alors été associée à leur pouvoir de dissipation entropique ${ }^{8}$. Un ARN simple brin dans l'eau étant plus efficace (rapide) que les bases simples (notamment la pyrimidine) pour dissiper par refroidissement vibratoire l'énergie d'excitation de photons UV absorbés directement. Ce qui en a fait émerger des systèmes dissipatifs d'énergie plus "performants" que l'absorption énergétique par le seul milieu aquatique.

Un modèle évolutif de diversification d'un ARNt monophylétique (Lacey et Staves, 1990) pourrait expliquer l'évolution ayant donné naissance au système de corrélation entre anticodons et acides aminés au sein des ARNt. En liant des segments courts de codons, une génération d'ARNm ancestraux, des pré-ARNm spécifiques auraient été créés étape par étape par les pré-ARNt pour le stockage de relations avec les séquences d'acides aminés d'ARNm (Chatterjee et YadavIci, 2016). Il apparaîtrait en effet que les acides aminés dont les caractéristiques sont les plus pertinentes pour favoriser la dissipation des photons UVC sont précisément ceux qui ont la plus grande affinité chimique pour leurs codons ou anticodons (Meija et Michaelian, 2018).

Les couplages d'acides ribonucléiques avec des combinaisons protéiques d'acides aminés ont ensuite progressivement donné naissance à des molécules plus efficaces encore sur le plan de la dissipation?

\footnotetext{
7 "Un système autopoïétique est organisé comme un réseau de processus de production de composants qui (a) régénèrent continuellement par leurs transformations et leurs interactions le réseau qui les a produits, et qui (b) constituent le système en tant qu'unité concrète dans l'espace où il existe, en spécifiant le domaine topologique où il se réalise comme réseau."

${ }^{8}$ Les isomérisations et les tautomérisations à l'état stationnaire induites par les UV-C et les UV-B se sont avérées cruciales pour la formation de purines à partir d'acide cyanhydrique dans un environnement aqueux, et pour la phosphorylation de nucléosides (Chatterjee et Yadavlci, 2018). Mc Reynolds et al. (1971) ont ainsi montré que des oligonucléotides peuvent ainsi être produits par l'action de la lumière ultraviolette sur une solution aqueuse de phosphate de nucléosides.

${ }^{9}$ L'alternance continue entre la polymérisation et dépolymérisation du cytosquelette notamment fournit une condition dynamique qui, couplée aux dipôles électriques, pourrait déterminer un mécanisme de dissipation de l'énergie électro-
} 
Cela favorisera par une augmentation de leur complexité l'émergence de phénomènes de reproduction des cycles et l'évolution de ces micro-organisations organo-moléculaires dissipatives vers des états plus résilients. Ceci notamment du fait d'un autre couplage, celui avec la reproduction par empreinte d'un de leur constituants fondamentaux, l'ARN dit messager puis, plus tard dans l'évolution, de celle, indépendante, des molécules-empreintes d'acide désoxyribonucléique (ADN) correspondantes, ceci avec un accroissement concomitant de la capacité dissipative des ensembles émergeants.

\section{5. "Ceci n'est pas (seulement) un code" : des "mystères de la vie" restent encore à élucider !}

Comme pour les recettes de cuisine, il faut les écrire une fois qu'elles sont établies et les cuisiniers sont alors encore en mesure de les adapter et de les faire évoluer par certaines variations en fonction à la fois des denrées et ressources disponibles et de l'évolution des attentes et exigences de la "clientèle". Si les recettes de cuisine une fois éprouvées s'écrivent pour en constituer le livre de leur mémoire, il est difficile d'imaginer dans une perspective systémique que c'est l'écriture aléatoire de telles recettes au sein d'un ouvrage qui ferait évoluer la gastronomie ... Et même, un restaurant ne passe pas du jour au lendemain de la cuisine italienne à la cuisine chinoise : il y a dans ce cas une vraie rupture, une vraie (r)évolution et pas de simples "variations" ou "adaptations".

Ce qui incite à mieux intégrer et relativiser, dans cette révision indispensable du paradigme de la nature des états vivants de la matière, les limites du pouvoir de chaque énoncé d'une recette, comme nous y incite par ailleurs l'émergence de la dimension "épigénétique" dans la description de ces états. il est devenu clair aux yeux des évolutionnistes que la redondance (sous la forme de copies multiples), présentée par de nombreux gènes, était peut-être un facteur crucial nécessaire à la complexification.

$\mathrm{Au}$ lieu de considérer que les propriétés de l'être vivant sont déterminées par le seul contenu moléculaire de l'ADN, nous savons aujourd'hui qu'il est question d'expression des gènes et non seulement de leur existence. En effet, tous les gènes de l'ADN sont inhibés au sein de la molécule et ne s'expriment que si leur inhibiteur épigénétique est désactivé.

En ce sens, ce qu'a essentiellement mis en évidence la génétique (néo)darwinienne jusqu'ici sont plus les mécanismes relativement linéaires, limités et continus de variation adaptative des systèmes biologiques comme les espèces animales ou végétales que ceux leurs réelles (ré)évolutions. Ce qui incite à devoir mieux intégrer et relativiser dans cette révision de la nature des processus d'évolution états vivants de la matière les limites de chaque "recette", comme nous y incite par ailleurs l'émergence de la dimension "épigénétique" dans la description de ces états. Les bouleversements évolutifs majeurs doivent en effet répondre au respect des principes d'organisation fondamentaux évoqués précédemment : dissipation, hétéro-organisation, seuils de rupture, co-détermination.

En particulier, lors des étapes majeures de l'évolution (cellules eucaryotes, pluricellularité, symbioses avec les systèmes bactériens différenciation des organes, évolution des systèmes nerveux, ...) il doit y avoir coévolution symbiotique des systèmes dissipatifs et de leurs systèmes de codage et de traduction de ces organisations dissipatives. Oserait-on dire que peut-être Jean-Baptiste Lamarck n'avait pas tout à fait tort ??

magnétique à la fois dans la gamme IR et dans la région visible (Pokorny, 2003). Ces interactions électrostatiques, comme dans les interactions dipôle-dipôle ou Coulomb, peuvent agir à longue distance et pourraient jouer un rôle dans l'organisation dynamique des premières réactions biomoléculaires dans la matière vivante. Les protéines chargées électriquement ont des moments dipolaires qui font qu'en effet elles pourront s'associer de 10 à 100 fois plus rapidement que ne le permet la simple diffusion brownienne (Stroppolo et al., 2001).

Dans ce contexte, Herbert Fröhlich, connu pour avoir donné la première explication de la supraconductivité résultant de l'interaction électron-phonon a développé l'idée que, comme dans une condensation de Bose-Einstein, les ondes de polarisation dans un systèmes polaire tel qu'une membrane cellulaire génèrent un champ électromagnétique connu sous le nom de "cohérence de Fröhlich" et, au-delà d'un certain seuil, le taux d'énergie provenant du milieu externe se concentrant dans le mode de fréquence vibratoire le plus bas, quasi toutes les composantes du système se synchronisent, c'est-à-dire oscillent de manière collective, à la fréquence la plus basse du spectre du système. Ce qui pourrait favoriser le transfert d'énergie à longue distance entre deux modes de vibration de fréquences différentes et ainsi dissiper des différences de quanta d'énergie. 
Il y a lieu de considérer notamment la co-détermination de la fonctionnalité de l'ARN messager et des ribosomes (reconnaissance de la boucle anticodon, décodage, biosynthèse des protéines), l'apparition au cours de l'évolution de nouvelles voies métaboliques pour les acides aminés et les nucléotides se développant simultanément (Wong, 2005), cette interaction entre les empreintes ("l'information") et ses structures de support fournissant les étapes logiques et incrémentales pour l'origine de la synthèse programmée de protéines marquent le début de l'évolution darwinienne. (Chatterjee et Yadalvci, 2018).

De ce point de vue, il est clair que le véritable code génétique ne réside donc pas, comme si souvent interprété, dans les "gènes", simples messages par combinaisons d'acides nucléiques, mais bien dans l'ensemble irréductible de la vingtaine d'ARN dits "de transfert", qui en constituent la grammaire, l'élément essentiel et réellement fondateur des "états vivants" de la matière. et dont chacun associe structurellement un triplet spécifique d'acides nucléiques ("codon") à un seul acide aminé. Cette famille irréductible et dynamique d'"hétéro-ARNs" forme ainsi au sein de son réceptacle cellulaire le véritable "moteur symbiotique hétéro-organisant" dynamique et (re)producteur de ces états vivants, leur "bouillon de culture" et dont l'ADN ne constitue alors "que" des empreintes.

Assemblés par ce bouillon, les acides aminés et autres "condiments" produisent ainsi la trame de protéines diverses aux propriétés catalytiques complémentaires à la fois dissipatives d'énergie et créatrice d'ordre dont la résilience des "messages" est assurée via la constitution de ses 'empreintes' au travers d'une syntaxe et d'une sémantique adaptées, le "matériel génétique".

Mais reste à établir comment les expériences du restaurant sont consignées dans le livre de recettes ... Et le point sur la question dépasse le cadre systémique, déjà (trop) large, de cet exposé et rejoint la question des relations entre sens et signe abordée lors d'un séminaire de l'AFSCET (de Gerlache, 2019a).

Notons simplement que les découvertes liées aux éléments de structure du matériel génétique tels la chromatine, le rôle des ADN dits "poubelle" (!) les ARN nucléaires, les processus de (dé)méthylation de l'ADN, recèlent sans doute ces mécanismes jusqu'ici incompris.

Comme celui qui consisterait pour le matériel génétique à constituer des "pages blanches" qui accumuleraient des "notes transitoires" transcrites par des procédés enzymatiques tels ceux des transcriptases inverses et autres télomérases. Au-delà de "seuils critiques", ces notes prendraient alors une forme cohérente dans leur nature hétéro-organisée pour favoriser l'émergence de nouvelles espèces d'états plus dissipatifs.

\section{3. "POUR-QUOI IL Y A DES ARBRES" OU L'ÉMERGENCE DES SYSTÈMES ORGANIQUES HÉTÉRO-REPRODUCTIBLES}

\subsection{L'émergence et l'évolution des systèmes dissipatifs multicellulaires : des états végétaux aux états animaux}

Les conclusions des travaux sur la structure de l'arbre de vie, loin d'être définitives, sont encore très controversées pour plusieurs raisons, notamment parce que, elles aussi bousculent violemment depuis deux décennies le modèle des trois domaines, le paradigme actuellement dominant (Aubert, Gupta, 1998 ; Lyons 2002; Forterre, 2005). Des endo-symbioses se sont alors produites entre certains organismes unicellulaires primitifs ayant une capacité de capture et de dissipation énergétique particulière, comme les chloroplastes et les mitochondries. Celles-ci ont alors ouvert la voie, avec la formation du noyau cellulaire, à l'émergence de structures pluricellulaires, encore plus efficaces et résilientes sur le plan dissipatif. Un peu comme on intègre une machine de production dans l'ensemble d'une usine et de ses services.

Il est généralement admis que la formation de grappes cellulaires, suivies du succès ou de l'échec de ces grappes en fonction de leurs caractéristiques, ont constitué les premières étapes vers la multicellularité. De nombreuses exigences d'organisation multicellulaire (adhésion cellulaire, communication et coordination cellule-cellule, mort cellulaire programmée) ont cependant probablement déjà évolué dans les organismes unicellulaires ancestraux. 
Le mouvement coordonné des cellules est le résultat de différents chemins de signalisation de cellule à cellule. La chimiotaxie explique notamment l'agrégation ondulatoire évoquée précédemment et observée in vitro (Goldbeter, 1996) qui détermine la dynamique spatio-temporelle de l'hétéroorganisation d'ensemble de cellules biologiques. La communication intercellulaire qui régit la transition de phases isolées à des phases collectives a notamment été montrée chez des moisissures comme Dictyostellum discoideumor qui émettent périodiquement un signal chimique pour attirer les cellules voisines.

À mesure que les grappes de cellules se sont adaptées, les cellules individuelles ont perdu leur autonomie évolutive, devenant des parties mutuellement dépendantes dans un nouvel ensemble de niveau supérieur. Ces transitions ont pu être facilitées par un processus symbiotique dans lequel les cellules adoptent des traits qui les ancrent dans un mode de vie de groupe, stabilisant le groupe et ouvrant la voie à l'évolution de la complexité multicellulaire (Les "cliquets" de Libby et Ratcliff, 2014).

Une étape cruciale de l'évolution biologique sera donc l'apparition des systèmes pluri- ou multicellulaires, au départ d'une segmentation d'une cellule unique (l' "œuf" ou ovocyte), dite phase de "blastula", prenant le pas sur la simple division cellulaire. Ce processus en est fondamentalement distinct en ce sens que se trouve constitué dans l'ensemble du "blanc d'œuf" épigénétique ainsi compartimenté un organisme global émergent. Ceci du fait de la répartition préalable au sein de "secteurs" distincts du cytoplasme de cette cellule "initiale" de différents facteurs spécifiques qui vont induire un potentiel de différenciation dans l'expression génétique de chacun des noyaux dupliqués. Cette caractéristique et ce rôle essentiel de l'organisation du cytoplasme de l'ovocyte ont été démontré dès le début des années 60 par John Gurdon (1962) et son équipe qui avaient réalisé l'expérience suivante :

a) prendre un œuf de crapaud fécondé ;

b) lui retirer son noyau ;

c) prélever sur un têtard albinos une cellule intestinale ou épidermique différenciée ;

d) transférer son noyau dans l'œuf énucléé.

Qu'observent-t-ils ? Non pas la formation de cellules intestinales ou épidermiques, mais le développement d'un embryon de têtard albinos : C'est le "blanc d'œuf" qui détermine et différencie ce que le génome du noyau transféré "exprime" ! ... Ce qui (dé)montre ici encore que l'origine des processus de différenciation tissulaire est, elle aussi une hétéro-organisation combinant mémoire épigénétique ou "cellulaire" et mémoire génétique.

Celle-ci se traduit notamment par le fait, bien établi lui aussi, que toutes les mitochondries d'un animal sexué, mâle ou femelle, sont d'origine maternelle, les mitochondries étant présentes dans l'ovocyte. Ou encore le processus de désactivation de l'un des deux chromosomes X chez la femelle fécondée qui est $100 \%$ épigénétique. Comme l'a montré Edith Heard, dans chaque tissu (le cerveau, le sang, les reins, etc.), la proportion de cellules qui activent le X paternel par rapport au X maternel peut être différente, et varie également d'un individu à l'autre. 


\section{Pour-quoi il y a des arbres}

Les végétaux multicellulaires, et les arbres en particulier, sont, particulièrement dissipatifs d'énergie via le processus d'évapotranspiration, la plante adaptant son activité photosynthétique à sa capacité de transport de l'eau mais aussi à la résistance thermique des feuilles aux réactions exothermiques engendrées par le métabolisme.

Elle favorise ainsi une évaporation optimale afin que le flux d'énergie net entrant dans le système soit presque entièrement dissipé dans l'atmosphère sous forme de flux de chaleur latente.

Dans ces végétaux plus complexes, les feuilles optimalisent l'évaporation, notamment par le biais de micro-valves présentes à la surface des feuilles appelées stomates. Un arbre peut de ce fait transpirer jusqu'à 220 litres/hr.

Ainsi, il est devenu possible de comprendre enfin que :

Tout autant un arbre se sert de l'eau pour croître, l'eau se sert de l'arbre pour s'évaporer !

L'évolution des états dissipatifs de la matière vivante vers des états de plus en plus complexes et efficaces s'est alors traduite par une diversification des systèmes caractérisés par une multiplicité d'espèces ou de "variants" aux rôles complémentaires, les éco-systèmes, contribuant à la résilience de ceux-ci, devenus plus tributaires des modifications de leur environnement.

Une étape particulière pour le règne végétal fut son acclimatation à la vie sur la terre ferme, la capacité dissipative offerte par la fonction photosynthétique s'y révélant beaucoup plus efficace.

\subsection{L'évolution de la symbiose biologique, entre efficacité et résilience}

Au-delà des systèmes végétaux, les systèmes animaux ont émergé eux aussi comme des systèmes de maximalisation de la dissipation de l'énergie, en l'occurrence, la dissipation de l'énergie accumulée par les organismes végétaux mais aussi celle accumulée par d'autres organismes animaux. Cela s'est traduit par la sophistication des états dissipatifs aux travers des multiples espèces, telle que la sophistication dans la différenciation des fonctions déjà observée chez les organismes végétaux.

En simplifiant à peine, les espèces animales ont évolué au travers d'une organisation de plus en plus sophistiquée autour d'un tube "digestif d'énergie" et la spécialisation des fonctions contribuant à maximaliser celle-ci au sein d'une organisation globale homogène:

- les cellules et leurs "organites" : noyau, mitochondries, lysosomes, membranes;

- les organismes et leurs "organes" : tube digestif, poumons, reins, foie, système musculaire, circulatoire, immunologique, systèmes hormonaux, lymphatique, , reproducteur, nerveux, ... ;

- les "sens", permettant d'optimaliser les interrelations tant spécifiques que systémiques avec le milieu extérieur et les autres êtres vivants ;

- les espèces et leur "diversité" : photosynthétiques, saprophytes, carnivores, y compris les espèces, jusqu'aux tribus et organisations socio-économiques humaines, et bientôt robotiques ...

- les écosystèmes combinant les espèces des différents niveaux d'organisation depuis le niveau monocellulaire, espèces qui seront sélectionnées en particulier du fait de leur contribution à des systèmes complexes d'équilibres dynamiques entre efficacité dissipative et résilience.

Un des véritables moteurs de l'évolution biologique est donc bien, outre la résilience ou "survie des plus aptes", tout autant une évolution vers des espèces et individus plus complexes quand cela les rend plus aptes ou efficace à la dissipation maximale d'énergie! On observe ainsi que, tel que l'a montré 
Eric Chaisson (2001, 2015), par unité de masse, le cerveau humain dissiperait jusqu'à 50.000 fois plus d'énergie que le soleil!

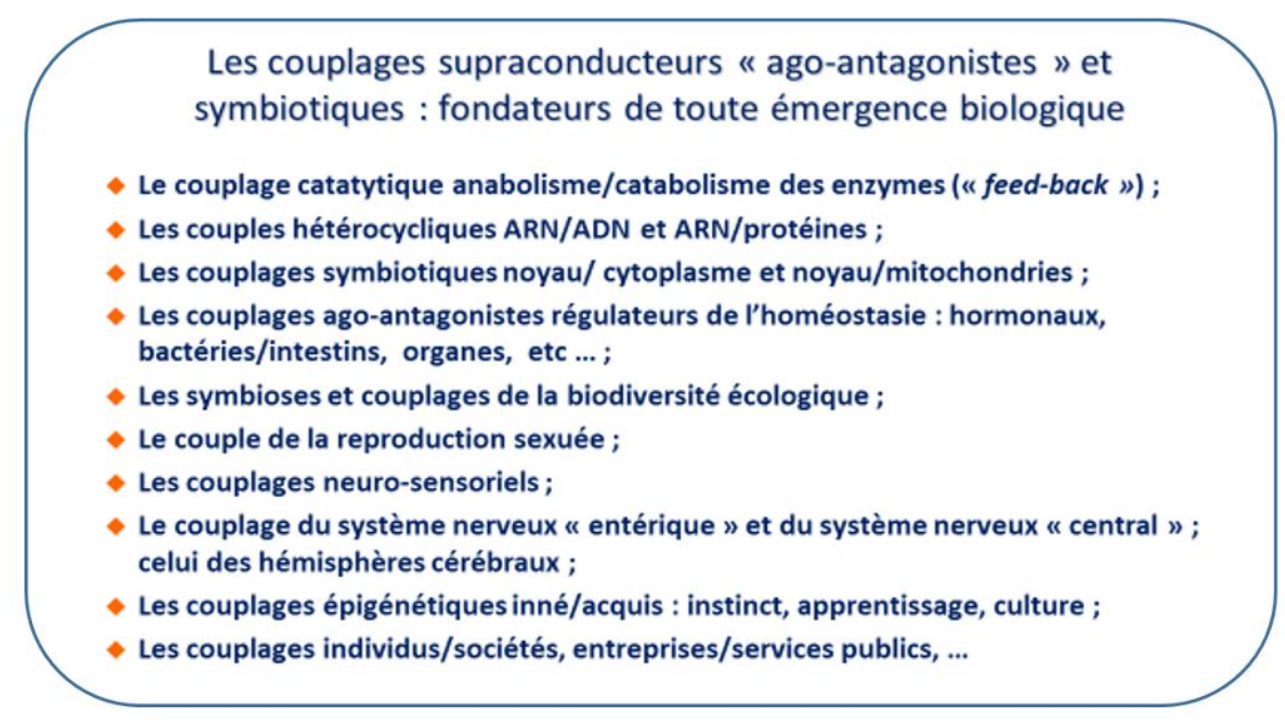

Une symbiose qui demeure fondamentale est celle entre les organismes unicellulaires et pluricellulaires s'organisant autour de la sophistication dissipative d'un système digestif. Comme tous les autres organes en contact avec le monde extérieur (peau, cavité buccale, oreilles, voies respiratoires supérieures, vagin) et délimitant l'espace intérieur d'un organisme, le système digestif est habité par des microorganismes unicellulaires avec lesquels il vit une symbiose efficace et vitale constituant ainsi de véritables écosystèmes, le "microbiote". Celui-ci est décrit dans toutes ses dimensions dans l'ouvrage de Marcel Roberfroid (2019) A la découverte du ventre et de ses bactéries. Une multitude de symbiontes associent en effet les organismes à des bactéries : la cavité buccale, les voies respiratoires supérieures, la peau, le vagin, les oreilles mais surtout le tube digestif avec l'intestin grêle et, davantage encore, le côlon. Un rôle majeur du microbiote colique est la protection vis à vis des dangers chimiques et microbiologiques qu'ils soient d'origine alimentaire ou environnementale.

En tant qu'interface entre le monde extérieur et l'intérieur d'un organisme, les deux 'tissus' vivent donc une symbiose étroite essentielle à la santé, tout en gardant, l'un de l'autre, une indépendance. Comme énoncé par l'écologiste microbien Théodore Rosebury cité par Marcel Roberfroid, "deux formes de vie peuvent établir des relations qui sont soit symbiotiques (et donc bénéfiques), soit parasitaires (et donc potentiellement dangereuses) en fonction du contexte". C'est le concept d'amphibiose proposé par qui rejoint celui des systèmes ago-antagonistes qui sera évoqué plus loin.

Toutes ces activités par essence symbiotiques doivent être coordonnées, contrôlées, régulées. Ainsi, le système digestif inclut aussi des glandes qui participent aux processus digestifs : les glandes salivaires, le pancréas exocrine ainsi que le foie et la vésicule biliaire ; quelques dizaines d'hormones sont aussi sécrétées par des cellules spécialisées du microbiome, les cellules L et K. Il est par ailleurs étroitement associé à un système nerveux intrinsèque et largement autonome, le système nerveux entérique ou SNE dont le mécanisme d'action est double : soit direct par liaison à une cible spécifique contrôlant un processus particulier; soit indirect, principalement par l'intermédiaire du nerf vague qui connecte directement les viscères avec le cerveau.

Comme suggéré par Susan Erdman, un axe microbiote - intestin - cerveau - système immunitaire pourrait exister et jouer un rôle clé pour la santé et selon l'expression de Min Li et coll. (2008, 2008), l'organisme, humain comme les autres organismes pluricellulaires, doit alors être considéré comme un 'super-organisme' résultant de cette association symbiotique avec un microbiote qui intègre des processus physiologiques propres (c'est à dire codés dans le génome humain) et ceux du microbiote (c'est-à-dire codés par le microbiome). 
La résilience d'un système biologique dépend donc de celle de chacun des composants: tout système (cellule, organe, organisme, espèce, (éco)systèmes, constitue un ensemble de chaînes trophiques en inter-actions hypercycliques solidaires (avantages) mais donc aussi moins autonomes (inconvénients). Une cellule, animale ou végétale, est déjà un écosystème formé par l'emboîtement et la juxtaposition de compartiments (les organites intra-cellulaires) qui forment une unité structurelle et fonctionnelle.

Les travaux conjoints de Robert Ulanowicz en écologie quantitative et de Bernard Lietaer en économie (2009) ont permis de (dé)montrer l'universalité de ce type d'équilibre dynamique vers lequel tendent alors l'ensemble des systèmes organisés, entre efficacité dissipative et résilience, ceci par la diversification des inter-connectivités, entre homéostasie et homéorhèse.

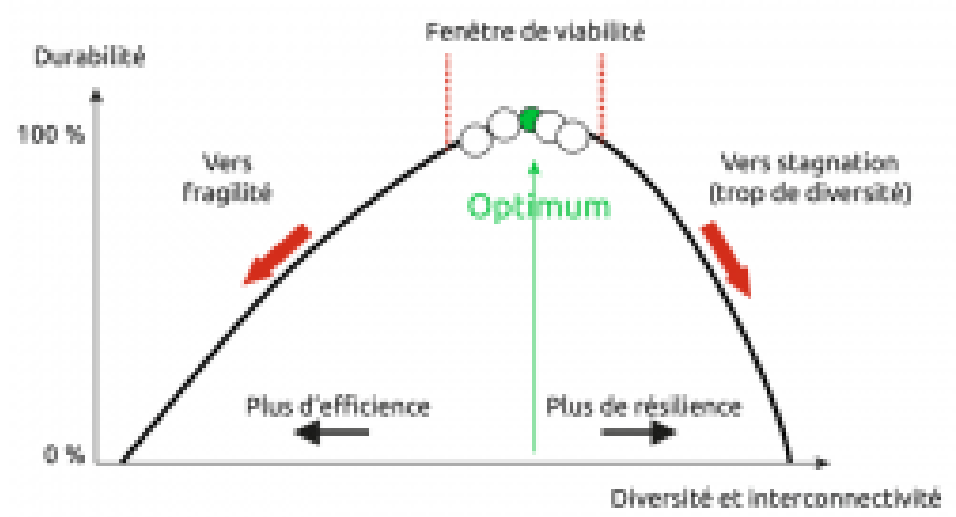

Source : Bernard Lietaer, Robert E. Ulanowicz, Sally J. Goerner, and Rocio Gomez (2009)

Ces états d'équilibres symbiotiques co-déterminés par les processus dynamiques décrits plus hauts évoluent donc dans le temps en fonction des contraintes thermodynamiques et environnementales et donc aussi épigénétiques : ce sont les phénomènes de croissance et de vieillissement.

Deux types de déviations décrits initialement par Conrad Waddington correspondent aux évolutions de tels systèmes dissipatifs organisés :

- les déviations inductives, résultant de la variation transitoire de facteurs physiologiques autour de l'état stationnaire dits d'homéostase; c'est l'image du mouvement latéral d'une bille sur les parois d'une rigole en pente ;

- les déviations constitutives dites d'homéorhèse, correspondant à une variation du mouvement vers l'équilibre final : c'est le mouvement de la bille vers le haut par rapport à la rigole (développement ou régénération) ou vers le bas (vieillissement).

Des états "pathologiques", comme par exemple les états cancéreux évoqués en introduction, sont à des états de groupes cellulaires échappant aux mécanismes de résilience du système symbiotique global (l'organisme"), ce qui correspond à des mouvements de la bille hors de la gorge.

L'homéorhèse et ses adaptations "constitutives" correspondent assez bien à la dimension agoniste du modèle de Bernard-Weil décrit plus loin, c'est-à-dire des phénomènes de coopération menant à la création de nouvelles solutions ("servo-mécanismes"), et non plus au seul "conflit" entre les forces antagonistes.

Toutes deux sont nécessaires pour que les systèmes dynamiques organisés soient à la fois capables de résilience et d'évolution adaptative : à la fois gérés par - et gérant des processus d'équilibre et de croissance. Ce type d'adaptation créative correspond aussi aux bifurcations se produisant en réponse à des fluctuations et permettant au système dissipatif de conserver un état stationnaire imposé par la contrainte thermodynamique globale du système au sein duquel il émerge.

Pierre Bricage (2013) a identifié dans ce contexte les cinq principes qui déterminent les conditions d'émergence de tels systèmes et leur permettent de maintenir un équilibre optimal entre résilience (ou 
stabilité) et efficacité dissipative maximale. Trop d'efficacité fragilise le système, trop de résilience en réduit l'efficacité par rapport à d'autres systèmes plus "compétitifs.

\section{les 5 principes organisateurs d'émergence constructale}

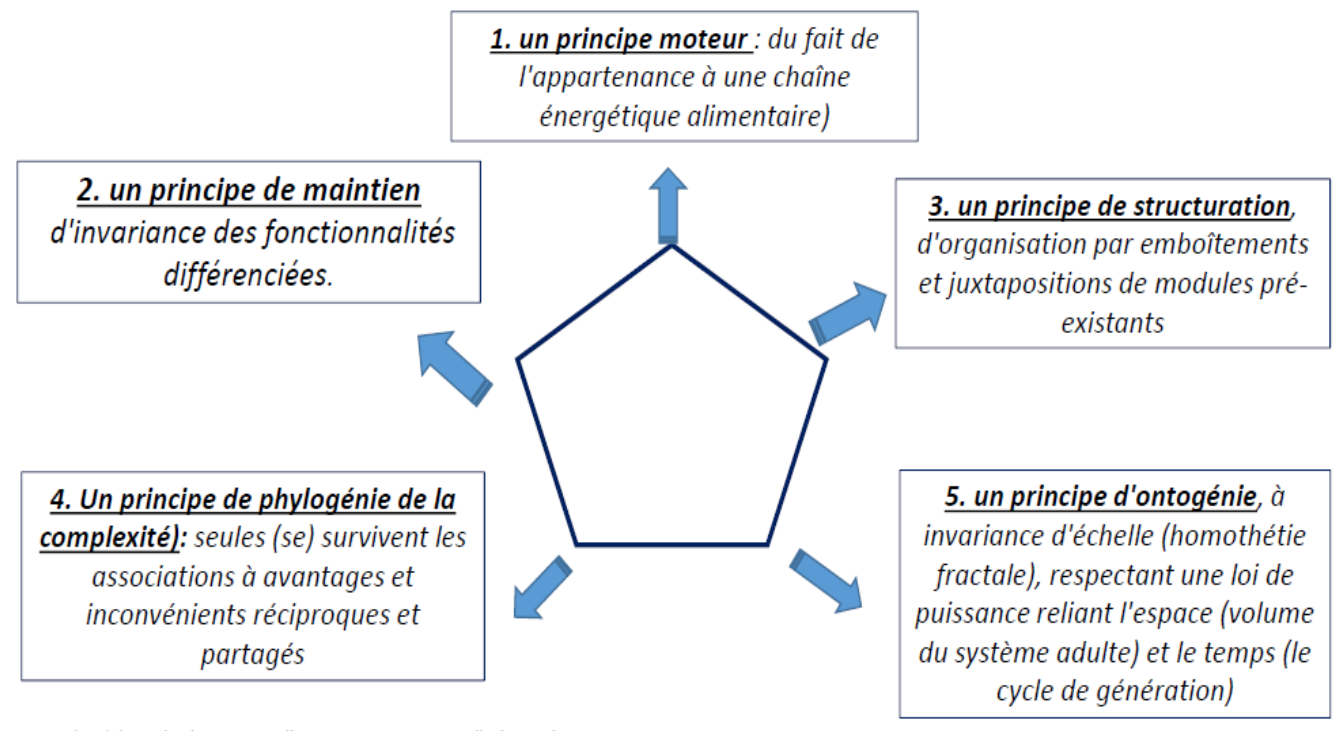

Adapté de : La loi de puissance d'invariance spatiotemporelle des systèmes vivants. Pierre Bricage -

Revista Internacional de Sistemas, 19, 05-33 2014

Les flux de matière et d'énergie qui le constituent y fonctionnent en flux tendus où les aliments des uns sont les déchets des autres et réciproquement. C'est ce que Pierre Bricage nomme des "Associations à Avantages et Inconvénients Réciproques et Partagés" ou "Associations for the Reciprocal and Mutual Sharing of Advantages and DisAdvantages" : ARMSADA.

Les organismes les plus complexes étant les plus "efficaces" du point de vue de la capacité dissipative mais, du fait de celle-ci, ils seront donc moins résilients face aux changements et perturbations écosystémiques. La disparition des dinosaures en est un exemple connu.

Dans un contexte de résilience, la sélection naturelle favorisera alors autant la "coopération" que la "compétition" :

- entre les gènes dans un génome ;

- entre les gènes et leurs produits/(re)producteurs (ARNs et protéines);

- entre organites d'une même cellule (chloroplastes, mitochondries, lysosomes) ;

- entre les cellules dans un organisme multicellulaire ;

- entre les individus dans une "société" (espèce) ;

- entre les espèces dans un écosystème.

François Roddier (2012) montre alors comment un (éco)système biologique évoluera en fonction de l'évolution biologique dans l'espace des phases :

- en fonction du taux de gènes communs et de leurs mutations ;

- mais aussi en fonction de l'évolution de l'environnement induite par les effets mêmes de la dissipation des systèmes qui imposent de toujours plus dissiper d'énergie pour maintenir leur état d'équilibre.

C'est effet dit de la "Reine rouge" d'Alice au Pays de Merveilles de Lewis Caroll: Alice court toujours plus vite pour pouvoir rester à la même place. 


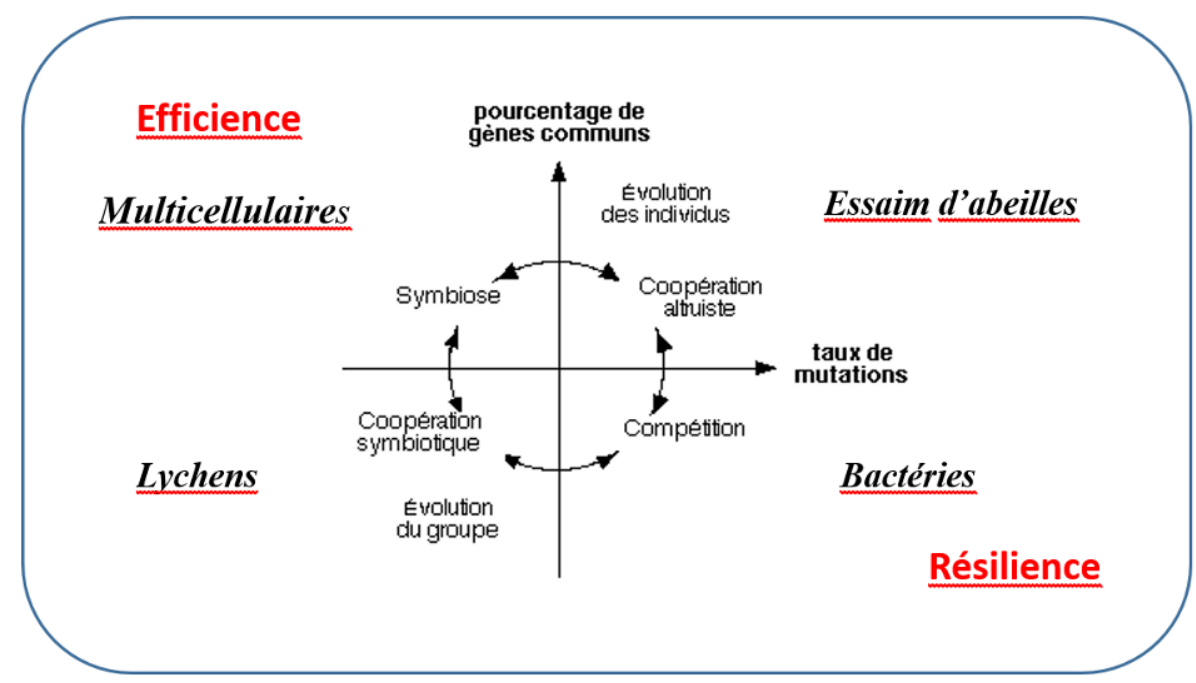

L'évolution biologique dans l'espace des phases, François Roddier

Avec le développement de la fonction cervicale ayant permis la diversification et la sophistication des "sens", ses processus s'élargiront au travers des capacités d'apprentissage et de mémorisation (épi)génétique : instincts animaux, conditionnement, éducation. Puis, au travers de l'émergence d'une capacité remémorisation volontaire transcendant la capacité de mémoire purement génétique et favorisant les états de "conscience" le développement de pratiques émotionnelles et "culturelles" : traditions, mythes et religions, pensée philosophique et artistique...

\subsection{Du concept biologique d'auto-organisation à celui d'hétéro-organisation en hypercycles ago-antagonistes}

Au travers du concept de co-détermination et de bi-pôle combinant celui qui fournit l'énergie avec celui qui en maximalise la "consommation" et donc la dissipation, il est possible d'évoquer, au-delà du concept-même d'"auto-organisation" et de "complexité", un processus d'hétéro-organisation, qui permet de l'affiner en y intégrant le fait qu'une telle organisation découle de la coexistence symbiotique intrinsèque en son sein de ces deux pôles et que tout système dynamique organisé est bien irréductible à une "causalité efficiente" unique.

\section{La notion d'hypercycle}

La notion d'hypercycle proposée par Manfred Eigen et Peter Schuster (1979) s'inscrit assez naturellement dans ce modèle d'hétéro-organisation, que ce soit pour décrire notamment les couples génotype - phénotype (les gènes, mais aussi les protéines et autres facteurs régulateurs de l'activité des gènes et des ARN messagers), tout comme les phénomènes hormonaux, immunologiques ou encore les processus dits "épigénétiques". L'ensemble des éléments d'un hypercycle sont de facto en mesure d'utiliser et/ou dissiper, plus efficacement que tout élément individuel, des ressources énergétiques existantes. En outre, les effets bénéfiques de mutations évolutives d'un élément quelconque de ces hypercycles sont alors répartis sur l'ensemble du système.

\section{Les couplages ago-antagonistes}

Dans ce contexte, Elie Bernard-Weil $(1988,1999,2002)$ a introduit la notion de couples agoantagonistes, couples de forces dont un des pôles produit des actions d'opposition sur une partie du récepteur de ces forces (antagonisme) et des actions de même sens sur une autre partie (agonisme). Celles-ci étant alors déterminées par :

- une norme d'antagonisme et des valeurs absolues assurant l'égalité du couple de forces, au moins quand le système est "au repos" ;

- une norme et des valeurs relatives agonistes prescrivant un certain degré d'intensité à ces forces en relation avec les circonstances. 
L'antagonisme déterminant des valeurs relatives, et l'agonisme des valeurs absolues de ces pôles de forces. C'est l'analyse de couplages hormonaux et de leurs perturbations qui est à l'origine de cette découverte importante. Il s'agit de modèles de croissance (ou de décroissance) et d'équilibre (homéostase) d'un état physiologique particulier localement hétéro-organisé autour de "normes" (équilibre surréno-post-hypophysaire, glycémie, par exemple).

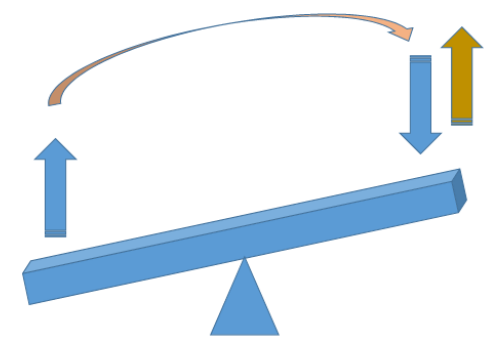

Si pour compenser un état pathologique (ex : certains cancers, AVC, immunopathies), un déséquilibre de la balance n'est compensé que par une augmentation de l'élément déficient, (flèche du côté gauche) le système va parfois "spontanément" compenser ce rééquilibrage (flèche de gauche du côté droit). Le rééquilibrage impose donc, paradoxalement d'aussi augmenter l'élément initialement en excès (flèche à l'extrême droite du côté droit).

Cette notion dépasse celle du classique couple d'opposition du clair et de l'obscur des présocratiques pour rejoindre celle du yin et yang qui n'est pas une simple opposition à laquelle on réduit trop souvent cette notion fondamentale du taoïsme...

L'existence des deux pôles distincts d'un couple AA implique à la fois l'absence d'un rapport de hiérarchie entre ces deux pôles ou de leur synthèse. Une action au niveau d'un seul sous-système globalement déséquilibré peut paradoxalement aboutir à la rééquilibration globale du système. même si ce type d'intervention sur deux forces apparemment inconciliables au sein d'un phénomène biologique est souvent considérée comme illogique, non seulement au plan biologique mais aussi au plan socio-économique ou politique.

Pour Elie Bernard-Weil, si le modèle ago-antagoniste est un modèle dissipatif, du type de ceux étudiés par Ilya Prigogine dans le cadre de la thermodynamique loin de l'équilibre, il en constitue un groupe à part, car il s'y établit des finalités d'équilibration par rapport à des normes : "Equilibration, c'est le résultat, tandis que "loin de l'équilibre" fait seulement allusion aux mécanismes par lesquels opère l'équilibration (aussi bien que la déséquilibration) qu'il s'agisse d'une équilibration asymptotique (fixe) ou sous forme de cycle-limite (oscillante) ou même d'une dynamique chaotique."

$\mathrm{Au}$-delà, les modèles élémentaires à deux pôles peuvent se combiner dans des réseaux AA, mais de telle façon que ces regroupements se fassent dans des structures associatives elles-mêmes AA Le modèle de régulation ago-antagoniste (AA) constitue donc un type de structure "fractale" voire "holographique" 10 que l'on peut retrouver à différents niveaux d'organisation d'un même système.

\subsection{Les causes co-déterminantes d'un phénomène dissipatif moléculaire : une synthèse}

Les causes co-déterminantes d'un phénomène dissipatif moléculaire sont donc identifiées :

- une tendance téléodynamique de réponse à la contrainte d'évolution du système global vers l'équilibre, cette "destinée" décrite par le second principe de la mécanique statistique, autre appellation de la thermodynamique ;

- un couplage spatio-temporel localisé de forces ago-antagonistes au sein du flux thermodynamique d'un milieu matériel loin de son équilibre et ayant ou acquérant la

\footnotetext{
${ }^{10}$ Dimension fractale ou holographique que, d'une certaine manière, l'on retrouve, comme le souligne notamment Edgar Morin, dans le fait que la plupart des cellules contiennent l'empreinte (via l'ADN et ses sous-structures complémentaires) de l'ensemble de la mémoire génétique de l'individu tout entier.
} 
propriété de catalyser localement des interactions spécifiquement dissipatives entre ses éléments constitutifs ;

- un système hétéro-organisé de supra-conduction dissipative qui résulte de cet équilibre dynamique intrinsèquement dual et symbiotique entre ses "causes co-déterminantes" ;

Ces bipôles ago-antagonistes couplés symbiotiquement au sein d'un système, comme dans le cas du cyclone avec des mécanismes de flexibilité associant à la fois des processus de compétition et de relations coopératives.

C'est à cette aune que doit être considérée la (co)détermination systémique, et, au-delà, il s'agit d'un point de vue plus général permettant d'échapper enfin au piège d'un déterminisme réduc-tueur. Cela permet ce qu'Edgar Morin nomme une dialogique ordre/désordre/organisation. Une telle dialogique permet d'assumer rationnellement l'inséparabilité de notions à priori contradictoires pour conce-voir un phénomène complexe: depuis la dualité onde/corpuscule jusqu'à celle du couplage individu/société. Cela permet aussi de (re)découvrir sous un autre éclairage la relation entre un tout et ses parties, particulièrement dans ls systèmes irréductiblement organisés en "sociétés. "Ills pensent, donc Je suis" : Mowgli demeuré seul dans la jungle aurait-il été pleinement "humain" ?

Cette co-détermination intrinsèque aux systèmes complexes organisés implique par ailleurs d'y intégrer les notions de flux et de champs de forces qui rendent compte, comme le souligne Jean-Louis Le Moigne (1999), de la complémentarité des changements intervenant sur ou dans un système dissipatif. A l'un, la physique associe les notions de tension (ou différence de potentiel), représentant le flux comme un écoulement entre une source et un puits, entre deux réservoirs (le "tout s'écoule" héraclitéen); à l'autre, elle associe la notion d'extensivité représentant les champs comme une orientation, une capacité d'influence, un tapis ordonné permanent de forces d'intensité variable.

Dans L'Homme symbiotique, Joël de Rosnay (1995), avait déjà élargi la théorie générale de l'autoorganisation à la notion plus générale de la dynamique de systèmes complexes et avait proposé le terme de symbionomie pour décrire l'ensemble des phénomènes couverts par cette théorie élargie dont on constate ici qu'ils correspondent aux états de résonance ago-antagoniste co-déterminés par les processus d'hétéro-organisation dissipative.

Quand, sur les traces de Claude Shannon, on tend à lier entropie et "quantité d'information", il serait sans doute pertinent de préciser que concrètement ce lien se situe, comme dans un circuit neuronal, au niveau de la nature et de la qualité des "interactions" entre éléments constitutifs du système considéré et dont émerge l'effet supraconducteur d'entropie. La probabilité de l'existence d'une information quantique peut être déduite du fait que des signaux instantanés induisent une cohérence dans les modèles diffractifs des mouvements des particules (Harris et Bush, 2013). Une "onde d'information" fonctionnellement liée à l'hétéro-organisation des propriétés dynamiques des particules en mouvement serait alors celle qui synchroniserait le mouvement énergétique d'une particule au moyen de flux de signaux oscillatoires et pas seulement une "fonction d'onde de probabilité". Tout ceci nécessiterait évidemment plus de développement ... 


\title{
4. TRANSCENDER ENFIN LES LIMITES DU PARADIGME RÉDUC-TUEUR DE LA SEULE "CAUSALITÉ EFFICIENTE"
}

\author{
"La description probabiliste est plus riche que la description \\ individuelle, qui pourtant a toujours été considérée comme la \\ description fondamentale. C'est la raison pour laquelle nous \\ obtiendrons au niveau des distributions de probabilités une \\ description dynamique nouvelle permettant de prédire \\ l'évolution de l'ensemble."
}

Ilya Prigogine

Il est donc établi que les phénomènes dynamiques d'hétéro-organisation dissipative s'observent aussi bien dans :

- les systèmes physiques et chimiques ;

- les systèmes cellulaires et organismiques : organisation énergétique, informationnelle, communicationnelle, reproductive, ...

- les (éco)systèmes végétaux : lichens, mousses, prairies, forêts, ...

- les (éco)systèmes animaux : organisation de fourmilières, de hordes, de troupeaux, de vols et migration d'oiseaux, ...)

- les systèmes et sociétés humaines : applaudissements, panique collective, manifestations, intentions de vote, cultures, religions, associations civiles et politiques, ...

- les systèmes sociétés socio-économiques :les entreprises, les réseaux urbains, industriels, commerciaux, ...

Mais, comme évoqué en introduction, en devenant plus réductionniste que réducteur, le paradigme analytique a limité la diffusion de ces connaissances pourtant essentielles à une meilleure compréhension de la réalité des phénomènes qui nous entourent et nous constituent. Il est si dommage que, comme le soulignait Max Planck, tant de chercheurs et d'individus mourront sans avoir bénéficié de ces lumières et sans les avoir intégré à leurs propres domaines de compétence (de Gerlache, 2015, 2016a, 2016b).

C'est la systémique de seconde génération qui, comme le souligne Gérard Donnadieu, en faisant le deuil de la prévisibilité (souvent impossible ou illusoire, considère-t-il) au profit de l'intelligibilité, permet de concevoir des modèles qualitatifs, de facture topologique par exemple, entrant dans l'intelligence d'un phénomène et d'orienter éventuellement son action. C'est de cette systémique "douce" que Jean-Louis Le Moigne cité par Donnadieu écrit en l'opposant à la première: "La modélisation analytique ou ensembliste se prêtait bien sans doute à l'appréhension des phénomènes compliqués, compliqués mais prévisibles; la modélisation systémique s'est développée précisément pour permettre ce passage réfléchi du compliqué au complexe, de la prévisibilité certaine à force de calcul à l'imprévisibilité essentielle et pourtant intelligible".

\subsection{Une dimension statistique dans la reproductibilité des organisations hétéro- organisées}

Comme le montrent de nombreux travaux de ce début de millénaire, et notamment les travaux de JeanJacques Kupiec (2012), les cellules peuvent se différencier spontanément lors d'événements aléatoires, les interactions cellulaires intervenant secondairement pour canaliser et stabiliser les cellules dans des états de différenciation adéquats. Il est erroné, nous dit Kupiec, de penser en particulier qu'un phénomène dépendant d'un événement aléatoire serait nécessairement non reproductible : il est reproductible statistiquement. "Ce que l'on met progressivement en évidence, ce n'est pas la spécificité des réactions sur le modèle instructif "clé-serrure" mais des paramètres quantifiables : les constantes cinétiques d'équilibre." "Un phénomène probabiliste peut être parfaitement reproductible si sa variabilité est très faible. Il y a de nombreux exemples en physique où les systèmes sont probabilistes 
au niveau de chaque molécule mais se comportent de manière déterministe au niveau macroscopique car le nombre de molécules impliquées est tellement grand que la variabilité globale du système devient négligeable."

Ces paramètres donnent une estimation de la vitesse d'association des molécules et de la stabilité du complexe qui en résulte. Toutes les molécules peuvent interagir entre elles avec des constantes cinétiques plus ou moins fortes : dans les cas extrêmes, la tendance à s'associer de certaines molécules est très forte et dans d'autres, très faible.

$\mathrm{Au}$ sujet de la reproductibilité globale et macroscopique d'un système, (cellulaire) malgré une variabilité microscopique (moléculaire), Kupiec évoque l'exemple imaginé par le biologiste Paul Weiss d'une plage fréquentée par des après-midis d'été :

- chaque après-midi, celle-ci sera caractérisée par une répartition des personnes dont la densité sera plus élevée au bord de l'eau, autour des restaurants ou d'autres attractions ;

- ce sera chaque jour la même chose et pourtant jamais deux fois ce ne seront exactement les mêmes gens aux mêmes endroits ;

- parfois, la prolifération est telle que la plage n'est plus organisée, comme dans le cas d'un cancer.

Paul Weiss montre que l'analogie n'est pas fortuite : les affinités entre les personnes, les corps hydrophobes au bord de la masse hydraulique, les distances entre les groupes l'attrait pour les nutriments, ... Cela ouvre de nouvelles perspectives sur les processus de développement et de différenciation en biologie: certains phénomènes dans les étapes de la croissance embryonnaire pourraient être déterminés par la reproductibilité statistique de certains états épigénétiques au cours des générations autant sinon plus que par l'expression "préprogrammée" de certains gènes ${ }^{11}$.

\subsection{Comment peut s'effondrer un système dissipatif éco-no-lo-gique hétéro-organisé}

L'équilibre dynamique d'un système dissipatif dépend donc de celui des éléments assurant la résilience de ses couplages hétéro-organisés. François Roddier a ainsi identifié les caractéristiques d'un mécanisme général de l'effondrement d'un écosystème, végétal, animal ou humain :

1. Il est associé à une grande fluctuation de son activité et il survient après une période de croissance inhabituelle de la taille de sa population ;

2. Une énergie disponible à faible coût provoque une augmentation de la température de

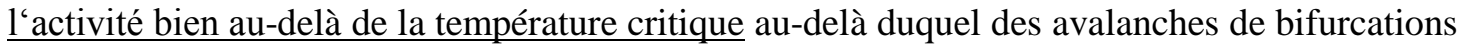
se produisent ;

3. La richesse en se condensant produit un accroissement des inégalités entre groupes au sein de l'écosystème ;

4. En raison du "toujours plus" ("effet de la Reine Rouge"), les écosystèmes parasites locaux doivent se réorganiser plus en plus vite en raison de l'accélération des changements qu'ils

${ }^{11}$ Des effets épigénétiques hérités sur les phénotypes ont été documentés chez les bactéries, les protistes, les champignons, les plantes et les animaux. Des exemples de transmission épigénétique transgénérationnelle induite par l'environnement chez les plantes ont également été rapportés et il existe des preuves préliminaires que ce mode de transmission est plus important chez les plantes que chez les animaux.

Le transfert d'expérience immunologique parentale pour améliorer la défense immunitaire de la progéniture est présent à la fois chez les vertébrés et les invertébrés, et peut être hérité pendant plusieurs générations. En outre, des preuves récentes suggèrent que des mécanismes épigénétiques tels que la méthylation de l'ADN, les modifications des histones et les petits ARN peuvent être à l'origine de bon nombre de ces réponses.

Un certain nombre d'études suggèrent aussi l'existence d'un héritage épigénétique transgénérationnel chez l'homme. Des facteurs et des mécanismes non génétiques sont connus pour être transférés du sperme au zygote, ce qui comprend la méthylation de l'ADN, les modifications des histones et le transfert de petits ARN et protéines. Non seulement les mécanismes épigénétiques transmettent des informations sur l'environnement, mais ils peuvent également être des acteurs importants dans le conflit sexuel entre les mères et les pères sur l'expression des gènes chez la progéniture. Les résultats suggèrent que les effets épigénétiques transgénérationnels paternels devraient être considérés comme des traits lourds et, par conséquent, développer une expression fortement dépendante des conditions dans lesquelles la sélection doit agir sur les effets épigénétiques transgénérationnels paternels. 
induisent eux-mêmes dans leur environnement par l'épuisement et (in)disponibilité des ressources ;

5. Progressivement la concurrence dans la reproduction (dite de sélection $\mathrm{r}$ ) l'emporte sur la coopération (dite de sélection $K^{12}$ ) favorisant la résilience sur l'efficacité ;

6. Un événement externe, même minime peut alors finalement déclencher leur l'effondrement, tel le flocon qui déclenche une avalanche ...

C'est dans ce contexte que peut être réévalué le processus de cancérogénèse. D'une manière générale la cancérogénèse est donc un processus lent, progressif et multiphasique dont la prolifération n'est qu'un aspect parmi d'autres. Aucune cellule réellement maligne n'est détectable dans les premières phases. L'autonomie acquise par les cellules cancéreuses implique une multitude de modifications qui s'expliquent assez mal par une altération ponctuelle. Si certains caractères de la malignité apparaissent dans de nombreuses cellules, seul un très petit nombre finit par se transformer complètement. En particulier, les foyers de cellules altérées perdent progressivement leur "appartenance sociale" et leurs fonctions " différenciées". La meilleure définition encore que l'on pouvait alors en proposer est celle formulée par Henri Pitot (1981) : "Le cancer est la croissance relativement autonome d'un tissu." En 1980, plusieurs phases distinctes étaient déjà distinguées dans la transformation maligne : la phase d'initiation : un événement impliquant une ou plusieurs altérations (épi)génétiques; elle est irréversible ou très prolongée mais rarement suffisante pour qu'un cancer se développe. S'en suit alors une phase de promotion des lésions initiales ; elle peut être réversible dans les premiers stades et les agents qui l'induisent ne sont pas nécessairement cancérogènes par eux-mêmes. Enfin, la phase de progression qui reflète l'expression d'une instabilité génomique ou la sélection clonale de cellules présentant des lésions supplémentaires.

Analysé, l'ensemble du processus a alors été décrit dans une perspective réellement systémique : L'analyse systémique du processus avait cependant déjà abouti à une description révisée, le concept R(DD)T (de Gerlache, Lans \& Roberfroid, 1986). Il consistait à décomposer le processus de cancérogénèse en quatre phases fonctionnelles distinctes mais combinées :

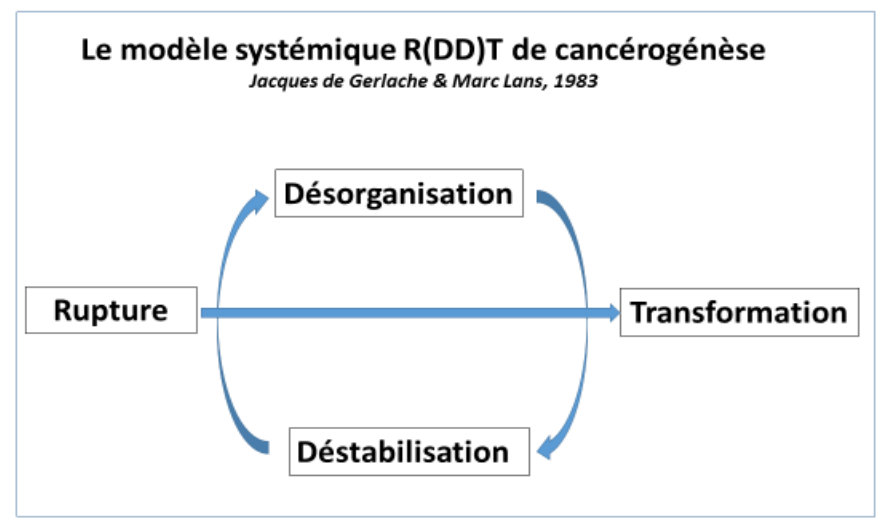

1. Rupture. Phase initiale induite par un agent cancérogène assimilée à une rupture des structures (épi)génétiques) responsables du maintien de l'homéorhèse, comme le couple mito-nucléaire CelIe-ci ne persiste que si il y a prolifération des cellules au moment de cette agression ;

2 et 3. Déstabilisation/Désorganisation. Ces effets combinés sont associés à la phase de croissance sélective et à une désorganisation progressive de l'état d'hétéro-organisation des cellules et du tissu altérés. Cette désorganisation favorise l'accentuation de la déstabilisation de l'équilibre cellulaire et homéorhésique. L'effet "promoteur" exerçé de façon chronique par certains agents ou états

${ }^{12}$ Voir çà ce sujet notamment : https://fr.wikipedia.org/wiki/Strat\%C3\%A9gie_de_reproduction 
physiologiques maintient cette désorganisation, épigénétique, ce qui amplifie la déstabilisation jusqu'à un point de rupture ;

4. Transformation: Les structures assurant le maintjen de l'homéorhêse sont altérées au point que les déviations constitutives ne sont plus suivies d'un retour à l'équilibre : en un point de rupture, certaines cellules sont libérées de ces contraintes et échappent au vieillissement et à la régulation de croissance. Un nouvel état autonomisé (la tumeur) s'établit et puise son énergie dissipative dans l'organisme dont elle s'est "autonomisée". Un peu comme un ghetto se constitue au sein d'une métropole urbaine ...

Cette description systémique de l'effondrement d'une structure dynamique organisée, celle d'un organisme différencié, anticipait ce que les observations sur l'importance des processus épigénétiques dans les processus tels ceux de la cancérogénèse allaient matérialiser dans les décennies qui suivirent, même si cette vision systémique ne reste encore que bien trop partielle et restrictive (de Gerlache, 2016c).

\section{EXEMPLES D'APPLICATION DES PRINCIPES DE L'HÉTÉRO- ORGANISATION SYSTÉMIQUE : DE LA GESTION D'UN ORCHESTRE A CELLE DES TRANSITIONS CLIMATIQUES ET ÉNERGÉTIQUES}

Dans une vision intégrative, l'enjeu de l'acceptation citoyenne devient aujourd'hui de plus en plus essentiel pour la mise en œuvre réussie des mesures d'infrastructure et des projets majeurs auxquels nos sociétés sont confrontées. Et ceci dans une période où la résurgence des visions simplistes portées par des individus, "charismatiques" fait redouter un retour aux formes de dictatures simplistes qui ont constitué la trame des tragédies du $\mathrm{XX}^{\mathrm{e}}$ siècle.

\subsection{La connaissance du solfège ne suffit pas à pouvoir coordonner un orchestre}

Le solfège est un peu la partie analytique de la musique : c'et le code qui permet de s'initier à la lecture précise d'une partition génétique nécessaire à son exécution : autrement dit, lire les empreintespartitions de la composition, formes équivalentes aux empreintes que sont des séquences d'ADN, couplées à différents "marqueurs épi-partitionnaires" comparables aux éléments de structure chromosomique et intégrée dans l'expression différenciée des partitions propres à chaque organeinstrument. Mais la connaissance du solfège ne fait pas un instrumentiste et la lecture d'une partition parmi des dizaines d'autres constituant la même œuvre, ne suffit pas à conduire l'exécution harmonieuse de $1^{\prime} œ u v r e$ par un ensemble de musiciens.

C'est que la musique elle-même est intrinsèquement systémique et hétéro-organisée au-delà du solfège et de l'apprentissage de l'instrumentation. Pour interpréter l'œuvre, Il faut aussi avoir appris la composition et l'orchestration dont elles sont les clés épi-partitionnaires. Hector Berlioz (1844) notamment a publié un Traité d'instrumentation et d'orchestration traitant de la composition musicale, considérée du point de vue des instruments et de l'orchestre. Il y affirme notamment que "l'orchestre peut être considéré comme un grand instrument capable de faire entendre à la fois ou successivement une multitude de sons de diverses natures, et dont la puissance est médiocre ou colossale, selon qu'il réunit la totalité ou une partie seulement des moyens d'exécution dont dispose la musique moderne, selon que ces moyens sont bien ou mal choisis et placés dans des conditions d'acoustique plus ou moins favorables".

Selon Henry Barraud, la trouvaille de génie de Berlioz porte sur cette distinction entre instrumentation et orchestration: "Instrumenter, c'est répartir au mieux, entre les instruments de l'orchestre, une musique préexistante. Orchestrer, c'est donner aux combinaisons des timbres, à leurs rapports et à leur succession, une valeur intrinsèque, c'est les faire entrer dans la composition, dans la conception même de l'œuvre comme un élément tout aussi essentiel, tout aussi organique que la mélodie, l'harmonie et le rythme."

Le jeu d'un orchestre symphonique consiste donc à coordonner (ce qui ne se réduit pas à "diriger" autoritairement !) l'exécution harmonieuse en temps et en lieu d'un projet (l'interprétation d'une "œuvre") qui combine des parties prenantes" (compositeur(s), musiciens, accompagnateurs, organisateur(s), et, bien sûr, public), des processus et des moyens (composition, partitions, solfège, 
instrumentation et jeu, orchestration, lieu) en fonction d'une interprétation, qui est l'ultime finalité d'un concert.

Si le milieu musical a été contraint de développer une approche et une méthode systémiques en mesure de relever tous ces défis, défis qui peuvent être sanctionnés dès les premières mesures de l'interprétation de l'œuvre - projet, ce n'est malheureusement pas le cas dans beaucoup d'autres projets de nos sociétés humaines complexes, si ce n'est, peut-être, dans certains domaines comme en matière militaire, médicale et aéronautique, pour ne citer que ceux-là. ... Avec l'évolution de nos sociétés, leur croissance démographique et technologique, celle de leurs besoins et de leur attentes, celle des contraintes matérielles (écologiques, socio-économiques, politiques, culturelles, morales et philosophiques), la nécessité est de plus en plus criante de coordonner dans un schéma stratégique général avec plus de rigueur (de gouvernance) et de méthode les parties prenantes, les enjeux et les contraintes de ces systèmes complexes.

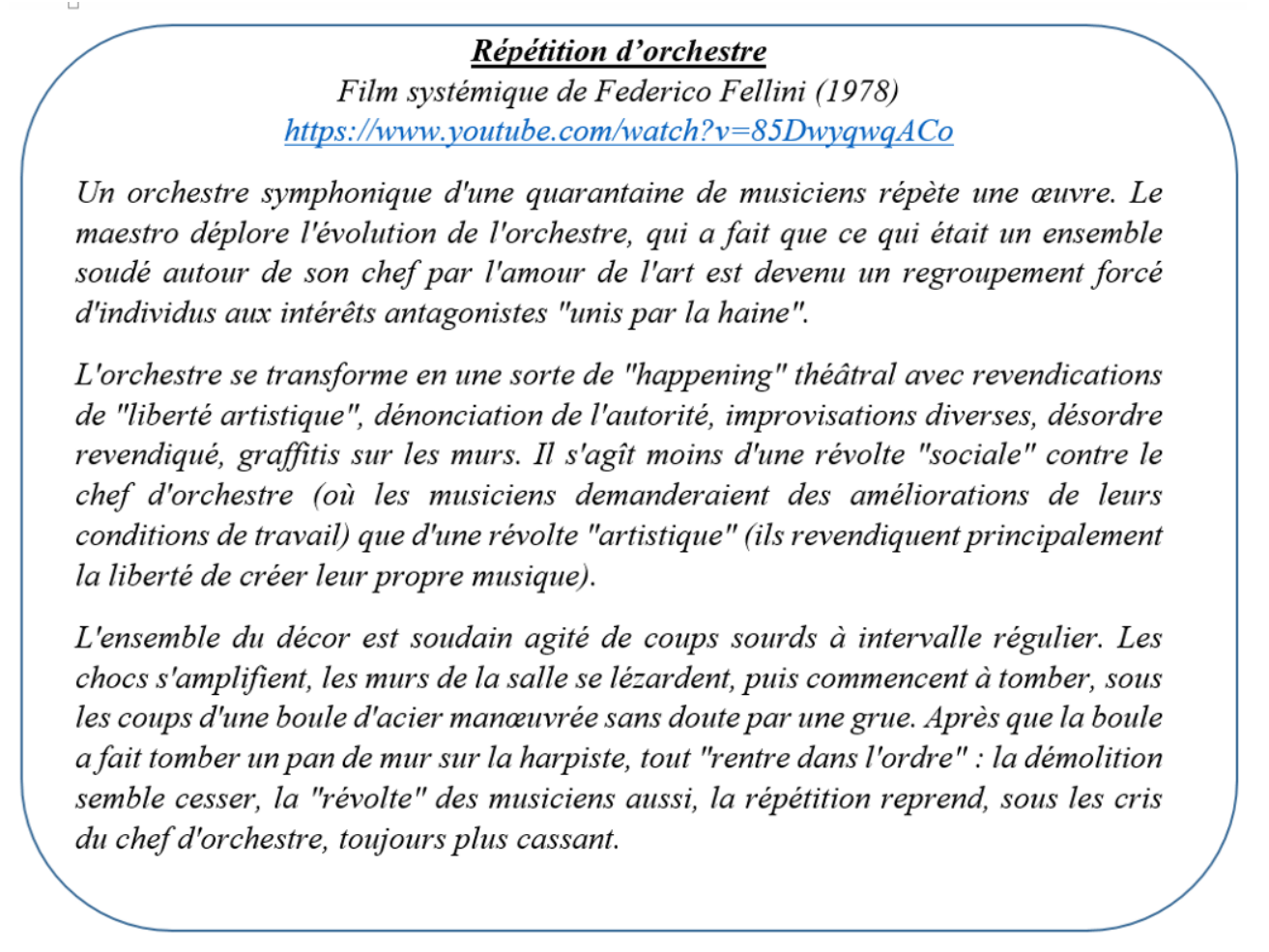

La plupart de ces défis ceux auxquels nos sociétés font face restent en effet abordés selon une approche trop fragmentée et trop réductrice, malgré les affirmations et les attentes de plus en plus insistantes de la nécessité d'approches intégrées pluridisciplinaires ou, mieux, transdisciplinaires. Il a été évoqué le fait qu'une raison de cette incapacité grave et préoccupante réside dans le fait que les méthodologies fiables d'analyse intégrative et systémique ne sont que trop rarement enseignées car elles sont considérées comme "secondaires" ou peu fiables et opérationnelles par ceux qui ne peuvent dépasser les limites du paradigme du "dogme réductionniste".

Face à la multitude des définitions, modèles, indicateurs, mais aussi colloques et littératures concernant la complexité, saisi du vertige que suscite le flou de ses contours, dans la plupart des cas, le technicien, le citoyen, ou le décideur politique, tous pétris d'une approche dissociative et réductrice des problèmes, sont le plus souvent dans l'incapacité de réellement intégrer ces différentes dimensions. Cela provient du fait que les outils pourtant disponibles et ayant déjà fait leurs preuves et permettent d'établir une vision globalisante mais rigoureuse de l'ensemble des enjeux dans leurs interactions, pour certaines intrinsèquement irréductibles, sont généralement considérés comme peu ou 
pas opérationnels parce qu'ils continuent à en ignorer l'existence alors qu'elles pourraient être appliquées si on prenait la peine de les pratiquer !

\subsection{Une perspective opérationnelle plus intégrative, entre dimensions analytique et systémique}

C'est dans ce contexte que de nombreuses méthodes-cadres systémiques existantes permettraient d'élaborer des projets échappant plus radicalement aux limites des modèles réducteurs et inopérants et d'aborder opérationnellement dans toute leur complexité l'ensemble des enjeux liés à la soutenabilité et au développement durable de leurs objectifs : causes efficientes et causes ultimes, (res)sources énergétiques, entités et organismes constitutifs, couplages organisationnels symbiotiques, enjeux et contraintes, efficacité, résilience, tout cela avec la lucidité d'en intégrer la dimension globale intrinsèquement dissipative. Ces modèles sont en particulier appliqués dans la gestion de l'organisation de la gestion des entreprises par des consultants spécialisés mais n'ont malheureusement pas diffusé dans l'ensemble des secteurs. Ceci en définissant et en articulant sans les borner, d'une part l'ensemble des attentes des différentes parties prenantes, et d'autre part les contraintes spécifiques à l'entreprise dans leurs interactions dynamiques et évolutives.

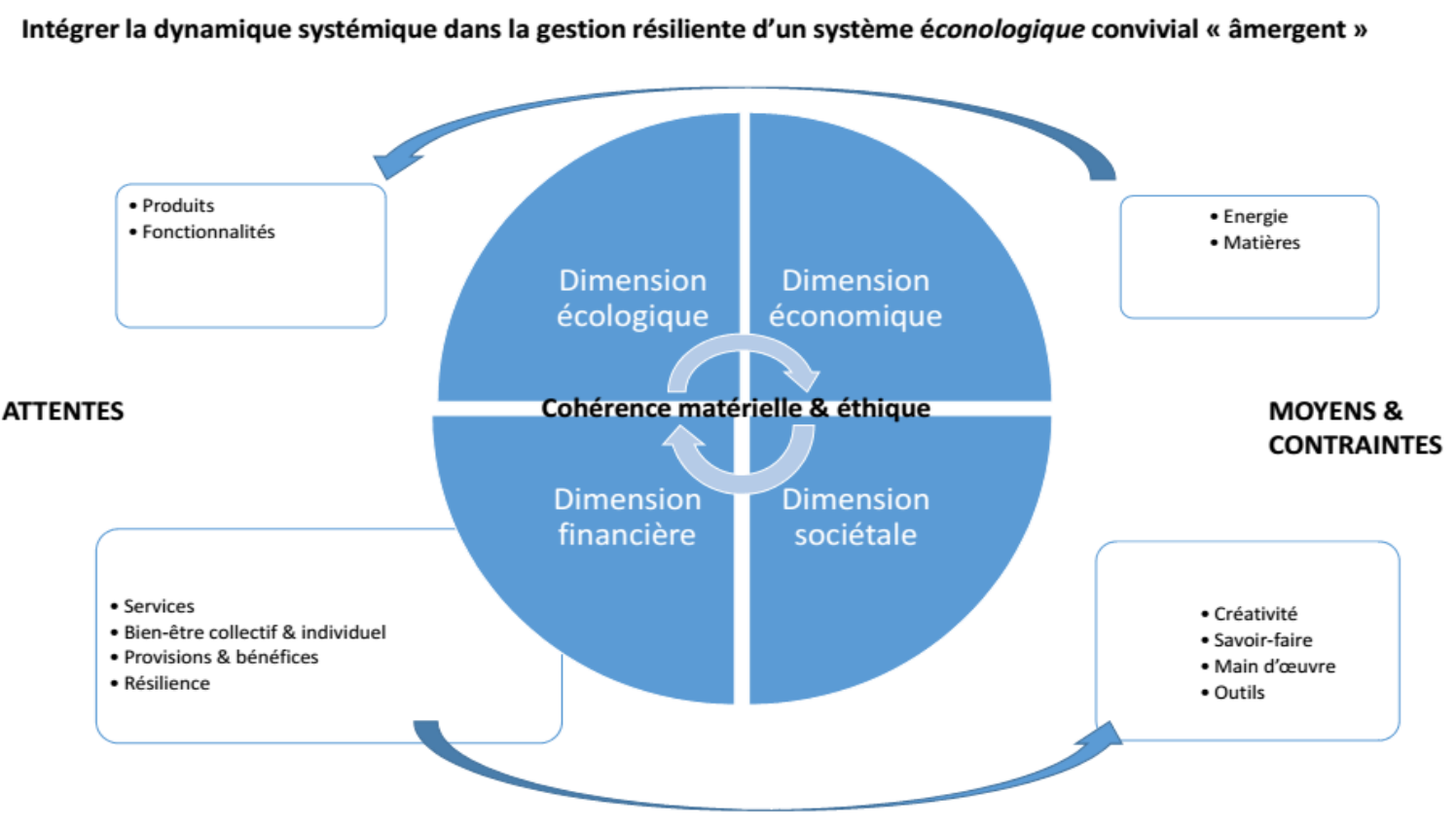

Un tel cadre élargi rend possible à chaque entité impliquée, chaque manager, d'analyser au sein de chacun des champs couvrant l'ensemble des enjeux potentiels, mais dans leur contexte global, ceux qui lui sont plus particulièrement pertinents.

Cependant, comme l'ont exprimé Bernard-Weil et aussi Eric Schwartz (2002), il n'y a aucun modèle "universel", qui permette de s'élever au-delà du niveau des modèles actuels est qui envisageraient de modéliser des propriétés intrinsèquement émergentes comme l'ensemble des modalités du comportement humain (et peut-être aussi de la nature) et celles issues de la liberté, de l'innovation, de la créativité et de soi-disant donner un accès à des prévisions radicales et certaines formes de décision. Ces devraient cesser de mobiliser les vains efforts d'une génération de chercheurs acharnés à prouver leur faisabilité, alors que leur seul résultat serait de détruire en fait de telles "facultés.

Des méthodes élargies à la gestion d'entreprises ou de "projets" dans leur ensemble ont déjà été élaborées qui intègrent alors non seulement l'ensemble de leurs collaborateurs mais aussi de leurs parties prenantes. Elles pourraient aujourd'hui mieux les articuler de manière symbiotique en y 
intégrant avec intelligibilité et lucidité les processus essentiels évoqués précédemment, tels les principes organisateurs d'émergence constructale et les processus ago-antagonistes.

Cette approche élargie, par essence-même plus collaborative que hiérarchique, permet à chaque acteur de se mobiliser et de juger avec clairvoyance du degré de prise en compte des contraintes de ses enjeux propres par rapport à l'ensemble de ceux de l'entreprise ou du projet. Il devient possible alors de fixer de façon beaucoup plus réaliste et opérationnelle le périmètre et l'orientation globale d'une stratégie qui intègre ces contexte propres à chaque acteur en y incorporant les diverses contraintes et attentes des autres acteurs et parties prenantes. Elle permet notamment de mieux prendre en compte les trois types de paramètres d'une éthique de gouvernance globale qui est indispensable à une bonne gestion de toute action sociétale : les paramètres politiques et réglementaires, les paramètres administratifs et les paramètres juridiques et d'en définir des indicateurs de suivi et de progrès très concrets.

\subsection{Développer une structure opérationnelle de projets symbiotiquement hétéro- organisée et intrinsèquement co-déterminée}

Ce cadre structurel hétéro-organisationnel élargi peut se matérialiser dans une approche matricielle qui permet de définir et d'expliciter les responsabilités de chaque partie prenante et de ses représentants ainsi que leurs interactions dans l'ensemble du "projet", ceci à partir des analyses, des données et des moyens disponibles ou mobilisables. Cette matrice intègre trois dimensions qui seront déclinées au départ d'un nombre maîtrisable de composantes :

- d'une part celles des attentes propres aux différentes parties prenantes ;

- d'autre part celles des enjeux spécifiques au(x) projet(s) considéré(s) ;

Ceci à la lumière d'une troisième dimension qui est celle des contraintes intrinsèques, génériques ou extrinsèques, y compris éthiques et de gouvernance, s'imposant aux deux autres dimensions.

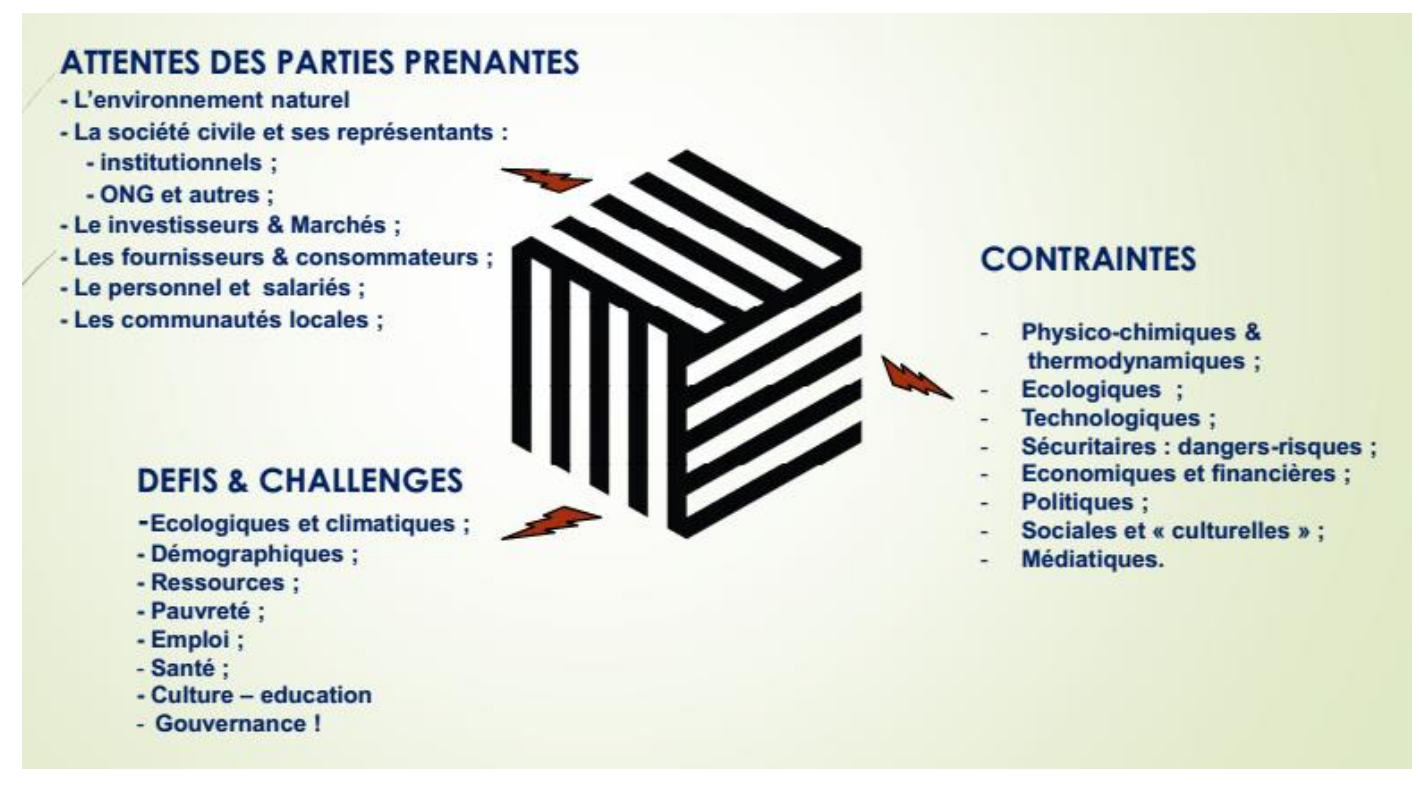

Une première matrice systémique bidimensionnelle, constituée de 16 à 25 champs d'action maximum pour pouvoir être "gérable", permet d'amorcer la démarche en matérialisant de façon simplifiée les principales parties prenantes et les principaux enjeux pour en établir les attentes et rôles respectifs, les "localiser" et déjà identifier interrelations et combinaisons qui en découleront, ceci à la lumière des contraintes génériques définies par la $3 \mathrm{e}$ dimension.

Une fois ces enjeux identifiés, clarifiés et articulés, leurs combinaisons co-déterminés peuvent s'intégrer en résonance avec l'ensemble des autres contraintes dans la perspective de la dynamique des 
objectifs globaux à atteindre mieux détaillés en matière. Ce qui ouvre la perspective d'une dynamique concrète, jusque-là paralysée par des démarches en "silos" et des horizons trop réducteurs. L'atout de cette démarche est donc de clarifier les interactions et les synergies entre ces différents enjeux et les responsabilités de chaque partie prenante, d'identifier le rôle dévolu à chacune par rapport aux autres intervenants et objectifs et ainsi pouvoir enfin assurer la cohérence d'ensemble de plans d'action dans l'espace et dans le temps à partir des analyses, des données et des moyens disponibles ou mobilisables et dont l'effet de résonance symbiotique ne pourra qu'en favoriser le succès. Cette méthode a déjà fait ses preuves dans la mise en œuvre d'une réelle politique de développement durable au sein d'une grand entreprise internationale en démontrant notamment son potentiel de motivation à atteindre des objectifs concrets et mesurés par des indicateurs appropriés.

\subsection{Application au cas des projets liés aux transitions des systèmes énergétiques}

Dans le cas des transitions énergétiques décidées dans le cadre de la maîtrise des bouleversements climatiques, parmi les paramètres essentiels à intégrer de façon systémique figurent de multiples enjeux et contraintes depuis la nécessité de l' efficacité énergétique, le poids des "externalités" climatiques et, plus largement, écologiques des (res)sources utilisées mais aussi des contraintes socioéconomiques et technologiques (extraction, ou production, transformation, stockage, transport, distribution, usages et recyclages, etc.) et, plus largement stratégiques et "politiques". Les externalités, notamment, ne sont encore trop souvent peu ou pas prises en compte et donc mal intégrées. Sans négliger d'autres problématiques associées et grandissantes elles aussi, comme la gestion socioéconomique et politique des réductions des ressources en eaux douces.

La restructuration ambitieuse du système d'approvisionnement énergétique se paralyse en effet sur nombre de ces enjeux comme en témoignent tragiquement toutes les Conferences Of the Parties (COP) des Nations-Unies, ceci en raison d'un manque d'acceptation, et donc menace d'échec, des objectifs nationaux et internationaux en matière de réduction des émissions de gaz à effet de serre.

Cette stratégie méthodologique est développée par ailleurs plus en détail (de Gerlache, 2019b) et une approche matricielle de ces enjeux de transitions énergétiques dans leurs différentes dimensions en a été esquissée.

Elle permet d'envisager plus concrètement comment enfin dépasser ici aussi une approche généralement trop fragmentée et trop réductrice de ces enjeux, de leurs contraintes et de leurs parties prenantes, ceci en réponse aux attentes de plus en plus insistantes, des générations à venir en particulier, de la nécessité, en cette matière comme dans bien d'autres, d'approches opérationnellement intégratives qui soient réellement symbiotiques et transdisciplinaires.

Dans ce contexte, il y a tant de problèmes sans solutions actuelles, selon Elie Bernard-Weil, tels 1'"entreprise" avec ses couples hiérarchie versus autonomie ou encore, stratégies émergentes vs stratégies délibérées, la socio-économie avec les couples collectivités territoriales vs autorité préfectorale, l'éducation $v s$ répression, la subsidiarité dans les États-nations européens vs institutions européennes, la mondialisation vs renforcement des identités locales, auxquels en intégrant la science des systèmes ago-antagonistes et la notion de non-modèle (notamment dans la démarche matricielle proposée ici), serait en mesure de proposer un début de solution optimale. Par exemple, l'intensification d'un conflit, à condition qu'elle soit équilibrée, est capable d'entraîner l'apparition de solutions jusque-là insoupçonnées. 


\begin{tabular}{|c|c|c|c|c|}
\hline & $\begin{array}{l}\text { Ressources énergétiques } \\
\text { Non émissives de } \mathrm{CO}_{2}\end{array}$ & $\begin{array}{l}\text { Transport de l'énergie et } \\
\text { son stockage (T\&S) }\end{array}$ & $\begin{array}{l}\text { Utilisations de } \\
\text { lénergie }\end{array}$ & Economies d'énergie \\
\hline $\begin{array}{l}\text { Acteurs } \\
\text { économiques et } \\
\text { financiers }\end{array}$ & $\begin{array}{l}\text { Développer des ressources } \\
\text { énergétiques adaptées aux } \\
\text { besoins industriels et } \\
\text { économiquement durables }\end{array}$ & $\begin{array}{l}\text { Développer des capacités } \\
\text { de Transport combiné et de } \\
\text { Stockage adaptées aux } \\
\text { besoins économiques }\end{array}$ & $\begin{array}{l}\text { Produire et offrir } \\
\text { P'énergie durable } \\
\text { nécessaire aux activités } \\
\text { industrielles }\end{array}$ & $\begin{array}{c}\text { Promouvoir des processus et } \\
\text { des produits réduisant les } \\
\text { besoins en énergie pour leur } \\
\text { production }\end{array}$ \\
\hline $\begin{array}{c}\text { Acteurs politiques } \\
\text { élus et } \\
\text { réglementaires }\end{array}$ & $\begin{array}{l}\text { Définir des systèmes de } \\
\text { taxation adaptés à la } \\
\text { nature et aux usages des } \\
\text { ressources énergétiques } \\
\text { utilisées }\end{array}$ & $\begin{array}{l}\text { Définir les besoins et les } \\
\text { règles concernant les } \\
\text { systèmes de gestion } \\
\text { assurant une répartition } \\
\text { équittable des différentes } \\
\text { formes d'énergie }\end{array}$ & $\begin{array}{l}\text { Proposer et imposer des } \\
\text { règles pour une gestion } \\
\text { durable et mieux intégrée } \\
\text { de l'ensemble des } \\
\text { dimensions du cycle } \\
\text { énergétique }\end{array}$ & $\begin{array}{l}\text { Définir des objectifs clairs et } \\
\text { des délais en ce qui concerne } \\
\text { le niveau d'efficacité } \\
\text { énergétique à atteindre }\end{array}$ \\
\hline $\begin{array}{l}\text { Citoyens et leurs } \\
\text { associations }\end{array}$ & $\begin{array}{l}\text { Assurer la disponibilité de } \\
\text { ressources énergétiques } \\
\text { durables adaptées aux } \\
\text { contraintes géophysiques } \\
\text { locales }\end{array}$ & $\begin{array}{l}\text { Favoriser les ressources } \\
\text { énergétiques locales et } \\
\text { développer les } T \& \text { en } \\
\text { respectant les attentes et } \\
\text { les besoins des citoyens }\end{array}$ & $\begin{array}{l}\text { Débattre sur les priorités } \\
\text { et les choix de société en } \\
\text { matière d'utilisation } \\
\text { d'énergie }\end{array}$ & $\begin{array}{c}\text { Développer des processus de } \\
\text { réductions significatives de la } \\
\text { consommation individuelle à } \\
\text { tous les niveaux }\end{array}$ \\
\hline $\begin{array}{c}\text { Acteurs } \\
\text { Soci(et)aux: } \\
\text { académiques, } \\
\text { éthiques, } \\
\text { culturels, ... }\end{array}$ & $\begin{array}{c}\text { Identifier et promouvoir la } \\
\text { combinaison de ressources } \\
\text { renouvelables la plus } \\
\text { durable et la plus efficace, } \\
\text { adaptée à chaque situation } \\
\text { locale dans son contexte } \\
\text { global }\end{array}$ & $\begin{array}{l}\text { Prendre en compte les } \\
\text { facteurs d'innovation } \\
\text { technologique, sociétaux et } \\
\text { environnementaux pour } \\
\text { améliorer la capacité et } \\
\text { I"efficacité des T \& S }\end{array}$ & $\begin{array}{l}\text { Rendre les citoyens et } \\
\text { leurs représentants } \\
\text { responsables de la } \\
\text { meilleure gestion } \\
\text { intégrée de leurs } \\
\text { utilisations d'énergie } \\
\text { durable }\end{array}$ & $\begin{array}{l}\text { Mobiliser les acteurs de la } \\
\text { société pour créer des } \\
\text { changements de paradigme } \\
\text { adaptés aux transitions aux } \\
\text { nouvelles ressources } \\
\text { énergétiques }\end{array}$ \\
\hline
\end{tabular}

Une matrice combinant les principaux enjeux liés aux transitions énergétiques avec les attentes des parties prenantes concernées https://www.dunod.com/sciences-techniques/mettre-en-oeuvre-transitions-energetiques-strategie-integrative-etgestion

\section{RÉFÉRENCES}

Aubert D. (2013). La transition bimembranées/unimembranées : une révolution au royaume des bactéeries?.<hal-01063767>

Belousov B.(1950) voir : https://en.wikipedia.org/wiki/Belousov\%E2\%80\%93Zhabotinsky reaction

Bénard H. (1901). Les tourbillons cellulaires dans une nappe liquide propageant de la chaleur par convection en régime permanent. Thèse de doctorat, défendue au Collège de France.

Berlioz H. (1844) Traité d'instrumentation et d'orchestration. Paris, Henry Lemoine, republié en 1993

Bernard-Weil, E. (1988) Précis de systémique ago-antagoniste, Introduction aux stratégies bi-latérales. L'Interdisciplinaire, Limonest .

Bernard-Weil E. ((2002) La Théorie des Systèmes Ago-Antagonistes., in Le Débat, Gallimard, Sept.Oct. 2002, pp. 106-119

Bernard-Weil, E. (2005) Les impasses stratégiques de la science des systèmes en biomédecine et socio-economico-politique sont en partie liées à la diffusion insuffisante des stratégies bipolaires comme à la non reconaissance du concept de non-modèle. Congrès Européen des Sciences des Systèmes. https://afscet.asso.fr/resSystemica/Paris05/bernardweil4.pdf

Bernard-Weil E. (2002) Stratégies Paradoxales en Bio-Médecine et Sciences Humaines. L'Harmattan, Paris, Budapest, Torino..

Bernard-Weil E. et Bricage P. (2002) Systèmes ago-antagonistes. Atelier 1 AFSCET https://www.afscet.asso.fr/6ESSC/Workshop01.pdf 
Bernard-Weil E. ( 2005) Le Non-modèle et ses modèles. http://www.homeolille.fr/wpcontent/uploads/2017/02/Bernard-Weil-.pdf ou http://plasticites-sciences-arts.org/PLASTIR/BernardWeil.pdf

Bricage P. (2013) Les Principes organisateurs d' émergence des systèmes vivants. GRECO Émergence et https://hal.archives-ouvertes.fr/hal-00830425/document

Chaisson, E.J. (2001) Cosmic Evolution: The Rise of Complexity in Nature. Harvard University Press: Cambridge, MA,

Chaisson E.J. (2015). Energy Flows in Low-Entropy Complex Systems Entropy 2015, 17, 8007-8018

Chatterjee S. (2016). A symbiotic view of the origin of life at a hydrothermal impact crater lake Physical Chemistry Chemical Physics 18(30)

Chatterjee S et Yadalvci S. (2018) The Origin of Prebiotic Information System in the Peptide/RNA World: A Simulation Model of the Evolution of Translation and the Genetic Code. Life 2019, 9, 25.

de Gerlache J. et Lans M. (1983). Analyse Systémique du Processus Multiphasique de la Cancérogénèse Hépatique. Concepts, Développement et Applications.Thèse de doctorat en Sciences pharmaceutiques Université de Louvain.

de Gerlache J, Lans M, Préat V, Taper H et Roberfroid M. (1984). Comparison of different models of rat liver carcinogenesis: conclusions from a systemic analysis.Toxicol Pathol. 1984;12, 4 374-382 http://tpx.sagepub.com/content/12/4/374.full.pdf

de Gerlache J., Lans M. et Roberfroid M. (1986). La cancérogénèse et la dynamique de l'organisation biologique: l'apport d'une description systémique dans l'analyse et la compréhension du processus. Auto-organisation et approche systémique des pratiques de recherche, Presses Universitaires de Namur. http://www.uclouvain.be/265407.html

de Gerlache J. (1994). Magritte meets Einstein: Encounter on the Road to the paradigm of Irreducibility. Conference proceedings "Einstein meets Magritte : An interdisciplinary reflection on science, nature, human action and society". Conference part of the 25th anniversary celebrations of the Vrije Universiteit Brussel - VUB http://pespmc1.vub.ac.be/Conf/einmagan.html

de Gerlache J. (2019). https://www.dunod.com/sciences-techniques/mettre-en-oeuvre-transitionsenergetiques-strategie-integrative-et-gestion

de Gerlache J. (2015). Une dimension réellement systémique dans l'analyse de nos organisations complexes : une nécessité urgente et impérative. Séminaire Etopia "L'économie s'est-elle affranchie des lois de la nature ?" http://www.etopia.be/spip.php?page=imprimerLight\&id article=2965

de Gerlache J. (2016a). L'enjeu du passage de la connai-science à la connaissance : entre méconnaissance méthodologique, individualisme idéologique et refus de lucidité. ler Congrès Mondial de la Pensée complexe UNESCO -Paris -https://www.reseaucanope.fr/fileadmin/user_upload/Projets/pensee_complexe/gerlache_enjeu_passage_connaiscience_co nnaissance.pdf

de Gerlache J. (2016b). Structures dynamiques et collectives : quelques déterminants élémentaires de leurs (dys)fonctionnements, du biologique au sociétal. Atelier interdisciplinaire de l'Institut des Systèmes Complexes (ISC-PIF) sur "L'approche systèmes complexes des processus de déstructuration et de résilience sociale". Paris 26 mai 2016

de Gerlache J. (2016c) De la thermodynamique à la cancérogénèse, une approche systémique des relations symbiotiques dissipatives Groupe Emergence Paris, 8 février 2016

http://www.mountvernon.fr/Complexite et cancerogenese/Complexite et cancerogenese.pdf

de Gerlache J. (2019a) Entre sens et signe : le mode de résilience des systèmes de couplages symbioenergétiques hétéro-organisés :de la thermodynamique dissipative aux systèmes socio-culturels. Séminaire AFSCET - Moulin d'Andé. En cours de publication.

de Gerlache J. (2019b). Mettre en œuvre les transitions énergétiques - Stratégie intégrative et gestion opérationnelle. Editions Dunod. 
https://www.dunod.com/sciences-techniques/mettre-en-oeuvre-transitions-energetiques-strategieintegrative-et-gestion

de Rosnay J. (1995). L'Homme symbiotique. Editions du Seuil

Donnadieu G. et Karsky M. (2002). La Systémique, penser et agir dans la complexité. Editions Liaisons.

Donnadieu, G._(2004). Systémique ou science des sytèmes - Quelques repères historiques. www.groupebena.org/IMG/doc/Histoire_de_la_systemique.doc

Dewar R. (2003) Information theory explanation of the fluctuation theorem, maximum entropy production and self-organized criticality in non-equilibrium stationary states. Journal of Physics -A: Mathematical and General, 36, 3.

Eigen, M. (1971). Self organization of matter and the evolution of biological macromolecules. Naturwissenschaften 58, 465-523

Eigen M. et Schuster P. (1979) The hypercycle - A Principle of Natural Self-Organization. SpringerVerlag Eds Part A" Emergence of the hypercycle. : https://pdfs.semanticscholar.org/eefb/7127bbf856e30ef16980d01297b830d3c2d5.pdf $\quad$ and http://izt.ciens.ucv.ve/ecologia/Archivos/Tomas_references-II/articles/def/eigen_schusternaturwiss7C.pdf

Farber E. (1984) Chemical carcinogenesis: a current biological perspective. Carcinogenesis.5, pp.1-5

Forterre P, Gribaldo S, Brochier C. Luca (2005). À la recherche du plus proche ancêtre commun universel. Médecine/sciences ; $21: 860-865.290$

Georgescu-Roegen N. (1979). Demain la décroissance. Entropie, écologie, économie. Traduit et présenté par Jacques Grinevald et Ivo Rens. Paris, Sang de la Terre; $3^{e}$ édition revue 1995 Paris, Sang de la Terre et Ellébore, 2006.] texte disponible en ligne

Gupta RS (1998). Life's third domain (Archaea): an established fact or an endangered paradigm? Theor Popul Biol ; 54 : 91-104.

Gurdon, J. B. (1962). The developmental capacity of nuclei taken from intestinal epithelium cells of feeding tadpoles. Journal of Embryology and Experimental Morphology. 10: 622-640

Hanahan D. et Weinberg R.A. (2000). Hallmarks of Cancer. Cell, 100, 1 57-70.

Hanahan D. et Weinberg R.A. (2011). Hallmarks of Cancer: The Next Generation. Cell, 144, 5 646674

Harris, D.M. and Bush, J.W.M. (2013). The pilot-wave dynamics of walking droplets. Physics of Fluids 25: 091112-1-2.

Ho Mae-Wan (2012) Cancer an Epigenetic Disease. $\underline{\text { http://www.i- }}$ sis.org.uk/Cancer an Epigenetic Disease.php?printing=yes

Kauffman, S. A.; Johnsen, S (1991). "Co-Evolution to the Edge of Chaos: Coupled Fitness Landscapes, Poised States, and Co-Evolutionary Avalanches" (PDF). Journal of Theoretical Biology. 149 (4): 467-505

Kauffman, Stuart (1993). The Origins of Order: Self Organization and Selection in Evolution. Oxford University Press.

Kauffman, Stuart (1995). At Home in the Universe: The Search for Laws of Self-Organization and Complexity. Oxford University Press.

Kauffman, S. (2006). "Beyond reductionism: Reinventing The Sacred". Edge.com. Edge Foundation

Kleidon, A. et Lorenz, R.D., (2005). Non-equilibrium Thermodynamics and Production of Entropy. Springer EDS: Berlin.

Kupiec J.J. (2012). L'ontophylogénèse. Editions Qwae

Lee D. et coll. (1997). Emergence of symbiosis in peptide self-replication through a hypercyclic network. Nature 390, 591-594, 
Lefever R., Nicolis G et Prigogine I. (1967). On the occurrence of oscillations around the steady state in systems of chemical reactions far from equilibrium. The Journal of Chemical Physics. 47: 10451047.

Le Moigne J.L. (1999) La modélisation des systèmes complexes. Editions Dunod.

Le Moigne J.L. (1994) Le constructivisme, Editions de L'Harmattan.

Li M. et coll (2008) Symbiotic gut microbes modulate human metabolic phenotypes. Proceedings of the National Academy of Science $105: 2117$.

Libby E. et Ratcliff W. (2014) Ratcheting the evolution of multicellularity.S cience 24: Vol. 346, 426427.

Lietaer B., Ulanowicz R., Goerner S.J. , and Gomez R. (2009) Quantifying Sustainability: Resilience, Efficiency and the Return of Information Theory. Ecological Economics, Vol. 6, Issue 1.

Lotka, A. J. (1922a). Contribution to the energetics of evolution. Proceedings of the National Academy of Science, 8, 147.

Lotka A. J. (1922b). Natural Selection as a Physical Principle. Proceedings of the National Academy of Science - 8, p 151 https://www.ncbi.nlm.nih.gov/pmc/articles/PMC1085053/pdf/pnas018910035.pdf

Lotka, A. (1925). Elements of Physical Biology (réimprimé en 1956 sous le titre : Elements of Mathematical Biology).

Lotka, A. J. (1945). The law of evolution as a maximal principle. Human Biology, 17(3), 167-196.,

Lyons SL. (2002) Thomas Kuhn is alive and well: the evolutionary relationships of simple life forma paradigm under siege ? Perspect. Biol. Med. ; 45 : 359-376.

Meija J et Michaelian K. (2019) Entropy Production as the Origin of Information Encoding in RNA and DNA. Preprints 2019090146 (doi: 10.20944/preprints201909.0146.v1).

Mejía, J. et Michaelian, K. Entropy Production as the Origin of Information Encoding in RNA and DNA. Preprints 2019, 2019090146 (doi: 10.20944/preprints201909.0146.v1). Copy

Michaelian K. (2011). Thermodynamic dissipation theory for the origin of life. Earth Syst. Dynam., 2, $37-51,2011$

Michaelian K. (2010). Thermodynamic Origin of Life. https://arxiv.org/pdf/0907.0042.pdf

Miller S. et Urey H. (1959) Organic Compound Synthesis on the Primitive Earth", Science, vol. 130, $\mathrm{n}^{\mathrm{o}} 3370$,

Monod, J. et Jacob, F. (1961). Teleonomic mechanisms in cellular metabolism, growth and differentiation Cold Spring Harb. Symp. on quant. Biol. 26, 389-401.

Per Bak. (1996) How Nature Works: the science of self-organized criticality, SpringerEds

Pokorný, J. 2003. Electromagnetic Biology and Medicine. Taylor and Francis, 22(1): 15-29.

Prigogine, I. (1969a). Structure, dissipation and life. In Theoretical Physics and Biology, ed. Marois, M.. Amsterdam: North Holland Publ. Co.

Prigogine, I., Lefever, R., Goldbeter, A. et Herschkowitz-Kaufman, M. (1969b). Symmetry-breaking instabilities in biological systems. Nature, Lond. 223, 913-916

Prigogine I. et Nicolis (1970) Biological order, structure and instabilities Quarterly Reviews of Biophysics Volume 4 Issue 2-3

Prigogine,I. (1980) From Being to Becoming: Time and Complexity in the Physical Sciences. San Francisco, W. H. Freeman \& Co.. Voir aussi : https://uplib.fr/wiki/Prigogine

Roberfroid M. (2019) A la découverte du ventre et de ses bactéries. Editions Josette Lyon. https://notre-siecle.com/a-la-decouverte-du-ventre-et-de-ses-bacteries-de-marcel-roberfroid-chez-

josette-lyon/

Roddier F. ( 2012), Thermodynamique de l'évolution - Un essai de thermo-bio-sociologie. Editions Parole 
Rubin H. (1992). Cancer development: the rise of epigenetics. European Journal of Cancer 28, 1-2

Schwartz, É. (2002) Can real life complex systems be interpreted with the usual dualist physical epistemology - Or is a holistic approach necessary?, 5th European Congress of Systems science

Steel E. J. Joël et coll. (2018) Cause of Cambrian Explosion - Terrestrial or Cosmic? Progress in Biophysics and Molecular Biology https://doi.org/10.1016/j.pbiomolbio.2018.03.004

Swenson, R. (1988). Emergence and the principle of maximum entropy production: Multilevel system theory, evolution, and nonequilibrium thermodynamics. Proceedings of the 32nd Annual Meeting of the ISGSR, 32, 32.

Swenson, R. (1989). Emergent attractors and the law of maximum entropy production: Foundations to a theory of general evolution. Systerm Research, 6, 187-197.

Turing A. (1952) The Chemical Basis of Morphogenesis. Philosophical Transactions of the Royal Society of London 237: 37-72.

Tyndall, J. 1869. Natural Philosophy in Easy Lessons - a physics book intended for use in secondary schools. Cassell, Petter \& Galpin, London.

Varela F. G, Maturana, F. H. et Uribe R. (1974). Autopoiesis: The organization of living systems, its characterization and a model. Biosystems, Vol. 5), p. 187-196.

Wicken, J. (1987). Thermodynamics, and Information: Extending the Darwinian Program. Oxford University Press.

Wiener N. (1948). Cybernetics or Control and communication in the Animal and the Machine, Hermann .

Wiener, N. Bigelow J. et Rosenblueth A (1987). Behavior, Purpose and Teleology. Republié par la Revue Internationale de Systémique, vol $1, \mathrm{~N}^{\circ} 1$.

Zhabotinski, A. M. (1964). Periodic course of oxidation of malonic acid in solution Biophysics 9, 306311. 